\title{
Role of conduction electrons in mediating exchange interactions in Mn-based Heusler alloys
}

\author{
E. Şaşığlu, ${ }^{1,2, *}$ L. M. Sandratskii, ${ }^{1, \dagger}$ and P. Bruno ${ }^{1, \sharp}$ \\ ${ }^{1}$ Max-Planck-Institut für Mikrostrukturphysik, Weinberg 2, D-06120 Halle, Germany \\ ${ }^{2}$ Institut für Festkörperforschung, Forschungszentrum Jülich, D-52425 Jülich, Germany
}

(Received 2 December 2007; revised manuscript received 18 January 2008; published 13 February 2008)

\begin{abstract}
Because of the large spatial separation of the Mn atoms in Heusler alloys $\left(d_{\mathrm{Mn}-\mathrm{Mn}}>4 \AA\right)$, the Mn $3 d$ states belonging to different atoms do not overlap considerably. Therefore, an indirect exchange interaction between $\mathrm{Mn}$ atoms should play a crucial role in the ferromagnetism of the systems. To study the nature of the ferromagnetism of various Mn-based semi- and full-Heusler alloys, we perform a systematic first-principles calculation of the exchange interactions in these materials. The calculation of the exchange parameters is based on the frozen-magnon approach. The Curie temperature is estimated within the mean-field approximation. The calculations show that the magnetism of the Mn-based Heusler alloys depends strongly on the number of conduction $s p$ electrons, their spin polarization, and the position of the unoccupied $\mathrm{Mn} 3 d$ states with respect to the Fermi level. Various magnetic phases are obtained depending on the combination of these characteristics. The magnetic phase diagram is determined at zero temperature. The results of the calculations are in good agreement with available experimental data. Anderson's $s$ - $d$ model is used to perform a qualitative analysis of the obtained results. The conditions leading to a diverse magnetic behavior are identified. If the spin polarization of the conduction electrons at the Fermi energy is large and the unoccupied Mn $3 d$ states lie well above the Fermi level, a Ruderman-Kittel-Kasuya-Yoshida-type ferromagnetic interaction is dominating. On the other hand, the contribution of the antiferromagnetic superexchange becomes important if unoccupied Mn $3 d$ states lie close to the Fermi energy. The resulting magnetic behavior depends on the competition of these two exchange mechanisms. The calculation results are in good correlation with the conclusions made on the basis of the Anderson $s-d$ model which provides useful framework for the analysis of the results of first-principles calculations and helps to formulate the conditions for high Curie temperature.
\end{abstract}

DOI: 10.1103/PhysRevB.77.064417

PACS number(s): 75.50.Cc, 75.30.Et, 71.15.Mb

\section{INTRODUCTION}

In recent years, Heusler alloys have become the subject of intensive experimental and theoretical investigations. The strong interest to these systems is mainly due to two unique properties: half-metallic behavior and martensitic phase transformations. ${ }^{1,2}$ The half-metallicity was first predicted by de Groot et al. in 1983 when studying the band structure of a half-Heusler alloy NiMnSb. ${ }^{3}$ Then, the half-metallic ferromagnets have become one of the most studied classes of materials. ${ }^{4-15}$ The existence of a gap in the minority-spin band structure leads to the $100 \%$ spin polarization of the electron states at the Fermi level and makes these systems attractive for applications in the emerging field of spintronics. Besides the strong spin polarization of the charge carriers, the half-metallic materials should have a crystal structure compatible with the industrially used zinc blende semiconductors and possess a high Curie temperature to allow the applications in the devices operating at room temperature. The available experimental information shows that Heusler alloys are promising materials also in this respect. ${ }^{16-19}$

At low temperatures, several Heusler compounds (i.e., $\mathrm{Ni}_{2} \mathrm{MnGa}$ and $\mathrm{Co}_{2} \mathrm{NbSn}$ ) undergo a structural transformation from a highly symmetric cubic austenitic phase to a low symmetry martensitic phase. The compounds that are magnetic in the martensitic phase can exhibit two unusual effects: magnetic shape memory (MSM) effect and inverse magnetocaloric effect. ${ }^{20,21}$ In MSM alloys, an external magnetic field can induce large strains when applied in the martensitic state. ${ }^{22}$ The MSM alloys are of great interest as promising smart materials for future technological applications. ${ }^{20,23-25}$ They can be used as sensors and actuators in different fields of applications. The inverse magnetocaloric effect (MCE) has its origin in the martensitic phase transformation that modifies the exchange interactions due to the change in the lattice characteristics. The inverse MCE effect was reported for samples with compositions close to $\mathrm{Ni}_{2} \mathrm{MnZ}(\mathrm{Z}=\mathrm{Ga}, \mathrm{Sn}) .^{21,26-29}$ In the martensitic phase, an adiabatic application of a magnetic field, rather than removal of the field as in ordinary MCE, causes the sample to cool. This feature is regarded promising for the development of economical and ecological refrigerants working near room temperature as an alternative to conventional vapor-cycle refrigeration.

Besides being promising materials for various applications, the Heusler alloys constitute a class of systems that is important also for fundamental researches. A wide diversity of magnetic properties makes Heusler alloys as critical test systems for the theoretical models of exchange interactions. Indeed, within the same family of alloys, one finds very different magnetic behaviors: itinerant and localized magnetism, ferrimagnetism, antiferromagnetism, helimagnetism, and other types of noncollinear ordering. ${ }^{30-36}$

Despite a key role of the magnetism in the properties of Heusler alloys, experimental and theoretical studies of the exchange interactions in Heusler alloys are still rare. The first important information on the exchange coupling in these systems was provided by the inelastic neutron scattering experiments of Noda and Ishikawa ${ }^{37}$ and Tajima et al. ${ }^{38}$ in the late 1970s. The authors measured the spin wave spectra of 
$\mathrm{Ni}_{2} \mathrm{MnSn}, \mathrm{Pd}_{2} \mathrm{MnSn}$, and $\mathrm{Cu}_{2} \mathrm{MnAl}$ for various directions in the Brillouin zone and analyzed the results of the measurements within the Heisenberg model. They obtained a long range and oscillatory behavior of the exchange interactions. The oscillations were reaching beyond the eight nearest neighbors in all three compounds. This behavior of the exchange interactions was considered as an evidence for an indirect exchange coupling, mediated via conduction electrons. The results were interpreted using either a RudermanKittel-Kasuya-Yoshida (RKKY) model or the $s$ - $d$ mixing model of Anderson (double resonance model). ${ }^{39-42}$ The double resonance model was found to be more relevant for Heusler alloys due to the strong mixing of the Mn $3 d$ states with the conduction electron states of nonmagnetic $3 d$ and $s p$ atoms.

This initial attempt of the theoretical analysis did not succeed in the description of the sign and the magnitude of exchange interactions between nearest and next nearest neighbors in contrast to good agreement for larger distances. ${ }^{43}$ The failure of the theories to describe nearneighbor exchange interactions was attributed to the asymptotic approximations. Price has shown that Anderson $s-d$ mixing model free of asymptotic approximations was able to capture qualitative features of the observed spin wave spectra of $\mathrm{Pd}_{2} \mathrm{MnSn}^{4}{ }^{44}$ Malmström et al. discussed the effect of the finite spin distribution around Mn atoms on the RKKY interactions. ${ }^{45}$ The authors showed that, in spite of the simplified treatment of the electron band structure, finite spin distribution could bring the calculated values of the exchange interactions in agreement with the exchange interactions determined for the $\mathrm{Ni}_{2} \mathrm{MnSn}$ and $\mathrm{Pd}_{2} \mathrm{MnSn}$ compounds from the experiments.

An important feature of the model Hamiltonian approaches is the possibility of separate study of different exchange mechanisms. However, the use of adjustable parameters and strong simplification of the band structure strongly restrict the possibility of reliable predictions for concrete materials. The development of the parameter-free density functional theory (DFT) has played a crucial role in the understanding of the physical properties of itinerant-electron ferromagnets.

The first contribution to the density functional theory of the exchange interactions and Curie temperature in Heusler alloys was made in an early paper by Kübler et al. where the microscopic mechanisms of the magnetism of these systems were discussed on the basis of the comparison of the ferromagnetic and antiferromagnetic configurations of the $\mathrm{Mn}$ moments. ${ }^{46}$ By analyzing the Mn $3 d$ density of states for different magnetic configurations, the authors proposed the mechanism of an indirect exchange coupling between Mn moments. Recently, the studies of the interatomic exchange interactions and Curie temperatures in Heusler compounds were reported by the present authors ${ }^{47-50}$ and Kurtulus et $a l .{ }^{51}$

\section{AIMS AND STRUCTURE OF THE PAPER}

Our previous studies on experimentally well established Ni-based compounds $\mathrm{Ni}_{2} \mathrm{Mn} Z(Z=\mathrm{Ga}, \mathrm{In}, \mathrm{Sn}, \mathrm{Sb})$ revealed a complex character of the magnetism in these systems. ${ }^{47}$ In particular, the obtained long range and oscillatory behavior of the exchange interactions as well as their strong dependence on the $s p$ atom $(Z)$ gave an evidence for the conduction electron mediated exchange interactions in Heusler alloys. The importance of the $s p$ electrons in the formation of the magnetic properties has been demonstrated by firstprinciples calculations also for a number of other systems. ${ }^{52-54}$ The experimental studies as well pointed out to the important role of the $s p$ electrons. The early measurements by Webster and Ramadan have shown this for quaternary Heusler alloys $\mathrm{Pd}_{2} \mathrm{MnIn}_{1-x} \mathrm{Sn}_{x}$ and $\mathrm{Pd}_{2} \mathrm{MnSn}_{1-x} \mathrm{Sb}_{x} .55$ Recent experimental studies on the Mn-based semi-Heusler compounds $\mathrm{Ni}_{1-x} \mathrm{Cu}_{x} \mathrm{MnSb}$ and $\mathrm{AuMnSn}_{1-x} \mathrm{Sb}_{x}$ revealed similarities in the behavior of the two systems with the variation of the number of valence electrons. ${ }^{56,57}$ In particular, the Curie temperatures of both systems decrease by the same amount with increasing concentration $x$ and both systems have similar $T_{C}$ values for compositions with equal numbers of valence electrons. These studies have shown that both the nonmagnetic $3 d$ atoms $(X)$ and the $s p$ atoms $(Z)$ play an important role in establishing magnetic properties. The explanation of the observed trends in magnetic characteristics of semi-Heusler alloys requires consideration of competing exchange mechanisms contributing to the formation of the magnetic state. One of the important aims of this work is to show that the results of the parameter-free DFT calculations for many systems can be qualitatively interpreted in terms of the competition of two exchange mechanisms.

In contrast to our previous studies, here, we go beyond the stoichiometric compositions and treat several Mn-based semi- and full-Heusler alloys within the virtual crystal approximation in order to understand the dependence of physical characteristics on the valance electron number.

The qualitative interpretation of the calculation results is based on the analysis of Anderson's $s-d$ mixing model. ${ }^{40}$ Since the atomic Mn moments are well defined, the application of the model is well founded. For the last four decades, the Anderson model has been successfully applied to a variety of problems of condensed matter physics and contributed to better understanding of numerous experimental properties. The systems studied within the Anderson model vary from dilute $3 d$ magnetic impurities in nonmagnetic metals ${ }^{58-61}$ to complex systems such as Heusler alloys, ${ }^{43-45}$ rare earths, ${ }^{62,63}$ magnetic multilayers, ${ }^{64-66}$ and diluted magnetic semiconductors. ${ }^{67-70}$

In the present paper, we show that the diversity of the magnetic behavior in Mn-based Heusler alloys can be interpreted in terms of the competition between ferromagnetic RKKY-type exchange and antiferromagnetic superexchange. A special attention is devoted to the role of the $s p$ and nonmagnetic $3 d$ atoms in mediating exchange interactions between $\mathrm{Mn}$ atoms. The spin polarization of the conduction electrons appears to be one of the key parameters in the formation of magnetic characteristics. We obtain a strong correlation between the spin polarization of the $s p$ electrons and the strength of the exchange interactions and, as a result, the value of the Curie temperature. It is shown that the position of unoccupied peaks of the Mn $3 d$ density of states plays an important role in determining the value of the anti- 


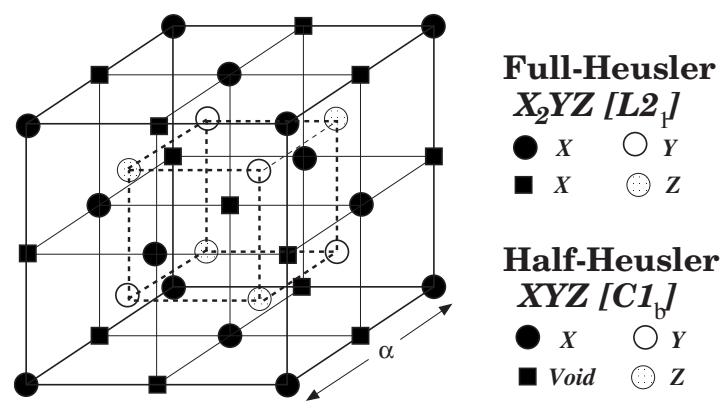

FIG. 1. $C 1_{b}$ and $L 2_{1}$ structures adapted by the half- and fullHeusler alloys. The lattice consists from four interpenetrating fcc lattices. In the case of the half-Heusler alloys $(X Y Z)$, one of the four sublattices is vacant. In VCA, the $Z$ site is occupied by a pseudoatom with a fractional number of valence electrons.

ferromagnetic superexchange coupling, while the properties of the conduction-electron states at the Fermi level are mainly responsible for the ferromagnetic RKKY-type exchange interaction. We compare the influence of nonmagnetic $3 d$ versus $4 d$ atoms on the exchange coupling by considering the stoichiometric $\mathrm{Ni}_{2} \mathrm{MnZ}$ and $\operatorname{Pd}_{2} \mathrm{MnZ}(Z$ $=\mathrm{In}, \mathrm{Sn}, \mathrm{Sb}, \mathrm{Te})$.

One of the main conclusions of the present treatment is that numerous features of the magnetism of the Mn-based semi- and full-Heusler alloys with different chemical compositions can be described with few parameters. Therefore, the tuning of these parameters can be considered as a tool in the fabrication of the materials with desired magnetic properties.

A part of the present work has been published elsewhere.$^{50}$ The remaining of the paper is organized as follows. In Sec. III, we present the details of the calculation approach. Section IV contains the discussion of the density of states and magnetic moments. In Sec. V, we dwell on the exchange coupling mechanisms and Sec. VI gives the conclusions.

\section{TECHNICAL DETAILS}

Semi- and full-Heusler alloys crystallize in the $C 1_{b}$ and $L 2_{1}$ structures, respectively (Fig. 1). The lattice consists from four interpenetrating fcc lattices. In the case of the semiHeusler alloys $(X Y Z)$, one of the four sublattices is vacant. The Bravais lattice is in both cases fcc.

The calculations are carried out with the augmented spherical wave method ${ }^{71}$ within the atomic-sphere approximation. ${ }^{72}$ The exchange-correlation potential is chosen in the generalized gradient approximation. ${ }^{73} \mathrm{~A}$ dense Brillouin zone sampling of $30 \times 30 \times 30$ is used. The radii of all atomic spheres are chosen equal. In the case of semiHeusler alloys, we introduce an empty sphere located at the unoccupied site.

We focus on the systems where the total magnetic moment is confined to Mn sublattice that simplifies the interpretation of the obtained results. To this end, we consider Pd and $\mathrm{Cu}$ containing Mn-based semi- and full-Heusler alloys that can be written in a compact form as follows:

$$
X_{k} \mathrm{MnZ}_{1-m} Z_{m}^{\prime} \Rightarrow\left\{\begin{array}{lll}
0 \leq m \leq 1, & k=1 & \text { for } \mathrm{s}-\mathrm{H} \\
0 \leq m \leq 1, & k=2 & \text { for } \mathrm{f}-\mathrm{H},
\end{array}\right.
$$

where $X=(\mathrm{Pd}, \mathrm{Cu}),\left(Z, Z^{\prime}\right)=(\mathrm{In}, \mathrm{Sn}),(\mathrm{Sn}, \mathrm{Sb}),(\mathrm{Sb}, \mathrm{Te})$, and $\mathrm{s}-\mathrm{H}$ (f-H) stands for semi-Heusler (full-Heusler) alloys. To account for noninteger electron numbers, we use the virtual crystal approximation (VCA) ${ }^{74}$ In VCA, the $Z$ site is occupied by In and Sn atoms according to their concentration which is described by an atom with fractional number of electrons $(1-m) z^{\mathrm{In}}+m z^{\mathrm{Sn}}$, where $z^{\mathrm{Sn}}$ is the number of electrons of Sn and similarly for other chemical elements. In this approximation, only the spin magnetic moment and the density of states (DOS) of the pseudoatom can be calculated. The properties of the pseudoatom cannot be projected on the constituting atoms. An advantage of VCA is the possibility of a continuous variation of the electron number without resorting to large supercells.

In the last column of Table I, we present the lattice parameters used in the calculations. The remaining columns give the lattice constants of the experimentally existing systems. Some of the compounds are not yet synthesized. For the synthesized systems, the alloys containing the $s p$ atom (Z) from the same row of the Periodic Table have similar values of lattice constants. Therefore, we use the average lattice parameters of the synthesized systems in the calculations for all $Z$ constituents. As seen from Table I, the difference between experimental lattice parameters and the parameters used in the calculations is less than $0.5 \%$.

To determine the interatomic exchange interactions, we use the frozen-magnon technique ${ }^{75}$ and map the results of the calculation of the total energy of the helical magnetic configurations, ${ }^{75,76}$

$$
\mathbf{s}_{n}=\left[\cos \left(\mathbf{q} \mathbf{R}_{n}\right) \sin \theta, \sin \left(\mathbf{q} \mathbf{R}_{n}\right) \sin \theta, \cos \theta\right],
$$

onto a classical Heisenberg Hamiltonian

TABLE I. Experimental and theoretical lattice parameters in $X_{k} \mathrm{MnZ} \quad(X=\mathrm{Pd}, \mathrm{Cu} ; k=1,2 ; Z$ $=\mathrm{In}, \mathrm{Sn}, \mathrm{Sb}, \mathrm{Te})$. For explanations, see text.

\begin{tabular}{lccccc}
\hline \hline Compound & $\begin{array}{c}a_{[\mathrm{In}]} \\
(\AA)\end{array}$ & $\begin{array}{c}a_{[\mathrm{Sn}]} \\
(\AA)\end{array}$ & $\begin{array}{c}a_{[\mathrm{Sb}]} \\
(\AA)\end{array}$ & $\begin{array}{c}a_{[\mathrm{Te}]} \\
(\AA)\end{array}$ & $\begin{array}{c}a_{[\mathrm{Z}]} \\
(\AA)\end{array}$ \\
\hline $\mathrm{PdMnZ}$ & & & 6.25 & 6.27 & 6.26 \\
$\mathrm{CuMnZ}$ & & & 6.09 & & 6.09 \\
$\mathrm{Pd}_{2} \mathrm{MnZ}$ & 6.37 & 6.38 & 6.41 & 6.38 \\
$\mathrm{Cu}_{2} \mathrm{MnZ}$ & 6.20 & 6.17 & & 6.18 \\
\hline \hline
\end{tabular}



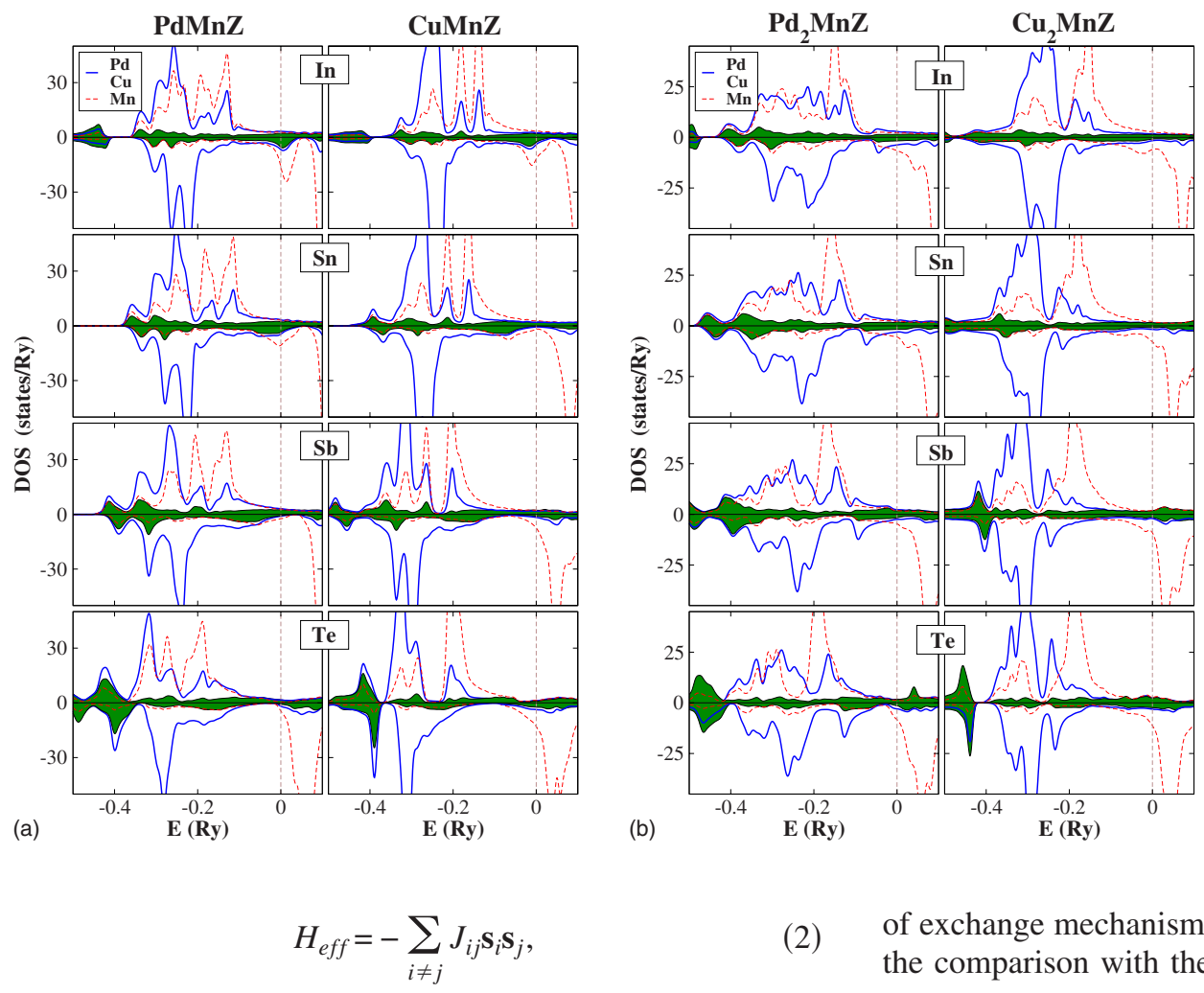

FIG. 2. (Color online) Left panel: Spin projected atomresolved density of states of $\operatorname{PdMnZ}$ and $\mathrm{CuMnZ} \quad(Z$ $=\mathrm{In}, \mathrm{Sn}, \mathrm{Sb}, \mathrm{Te})$ for stoichiometric compositions. The shadow areas show the DOS of the $Z$ constituent. The broken vertical lines denote the Fermi level. Right panel: The same for full-Heusler compounds $\mathrm{Pd}_{2} \mathrm{MnZ}$ and $\mathrm{Cu}_{2} \mathrm{MnZ}$. where $J_{i j}$ is an exchange interaction between two Mn sites, $\mathbf{s}_{i}$ is the unit vector pointing in the direction of the magnetic moment at site $i, \mathbf{R}_{n}$ are the lattice vectors, $\mathbf{q}$ is the wave vector of the helix, and $\theta$ is the polar angle giving the deviation of the moments from the $z$ axis. Within the Heisenberg model [Eq. (2)], the energy of frozen-magnon configurations can be represented in the form

$$
E(\theta, \mathbf{q})=E_{0}(\theta)-\sin ^{2} \theta J(\mathbf{q}),
$$

where $E_{0}$ does not depend on $\mathbf{q}$ and $J(\mathbf{q})$ is the Fourier transform of the parameters of interatomic exchange interactions,

$$
J(\mathbf{q})=\sum_{\mathbf{R}} J_{0 \mathbf{R}} \exp (i \mathbf{q} \cdot \mathbf{R}) .
$$

Calculating $E(\theta, \mathbf{q})$ for a regular $\mathbf{q}$ mesh in the Brillouin zone of the crystal and performing back Fourier transformation, one gets exchange parameters $J_{0 \mathbf{R}}$ between pairs of Mn atoms.

The Curie temperature is estimated within the mean-field approximation (MFA),

$$
k_{B} T_{C}^{M F A}=\frac{2}{3} \sum_{j \neq 0} J_{0 j} .
$$

\section{DENSITY OF STATES AND MAGNETIC MOMENTS}

The electronic and magnetic structures of Heusler alloys have been extensively studied earlier and the reader is referred to Ref. 4 and the references therein for a detailed overview. Here, we present a brief description of the calculation results aiming to provide the basis for the discussion of exchange mechanisms in subsequent sections and to allow the comparison with the previous work.

\section{A. Density of states}

In this section, we discuss the density of states for the stoichiometric compositions of both families of compounds [ $\left.X_{k} \mathrm{MnZ}(X=\mathrm{Pd}, \mathrm{Cu} ; k=1,2 ; \mathrm{Z}=\mathrm{In}, \mathrm{Sn}, \mathrm{Sb}, \mathrm{Te})\right]$. The results are presented in Fig. 2. The DOS for nonstoichiometric compositions assumes intermediate values. In agreement with the commonly accepted picture of the magnetism of the Mnbased Heusler alloys, we obtain a strong localization of the magnetization on the $\mathrm{Mn}$ sublattice with a value of the $\mathrm{Mn}$ moment close to $4 \mu_{B}$. In the following, we will show that the value of the Mn moment is very robust with respect to the variation of the magnetic structure. Therefore, we can conclude that Mn-based Heusler alloys possess a well-defined atomic Mn moment. The robust character of the Mn moment results from the large exchange splitting of the Mn $3 d$ states. It is important that the Mn $3 d$ states of only one spin projection (spin up) are strongly occupied. The main part of the spin-down Mn 3d states lies above the Fermi level.

The analysis of the DOS of different compounds reveals a relative shift of the Fermi level to a higher energy position in the sequence In-Sn-Sb-Te (Fig. 2) that results from the increasing number of valence electrons within this series. Indeed, a Te atom has three more valence electrons than In, two more electrons than $\mathrm{Sn}$, and one more electron than $\mathrm{Sb}$. The Mn DOS for semi-Heusler compounds shows an interesting property that different compositions give similar features if the total number of the $s p$ electrons coming from different atoms is the same. This similarity is more pronounced near the Fermi level. For example, the peaks in the Mn spin-down states of PdMnSn and CuMnIn are very similar to each other. As a consequence, the Mn atomic moments are also similar. This property can be related to the symmetry 

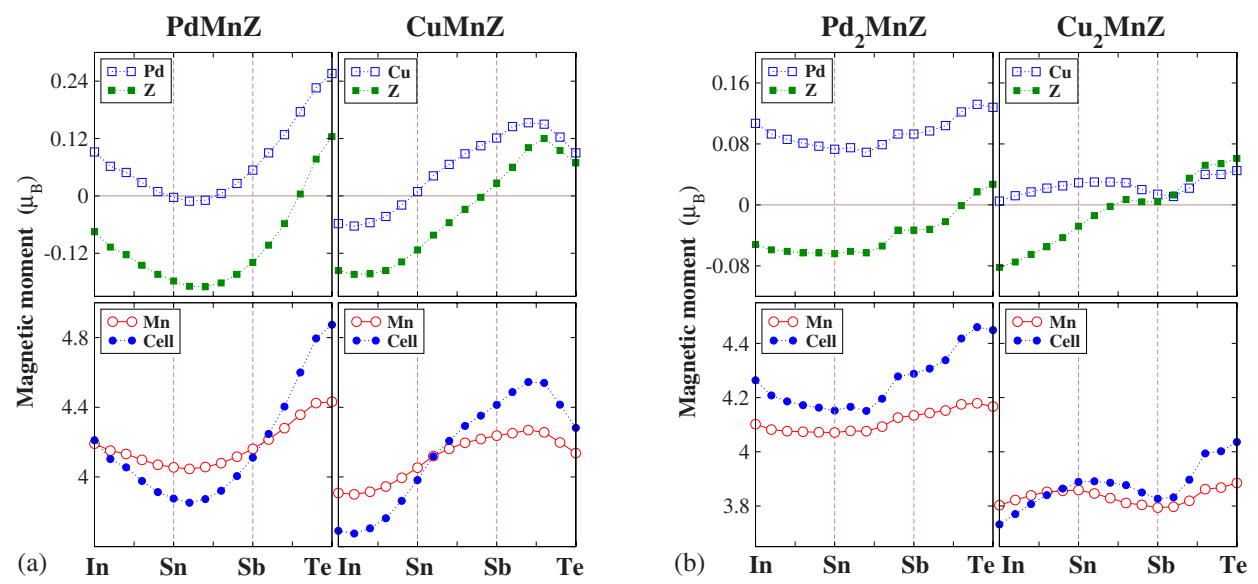

FIG. 3. (Color online) (a) Calculated atom-resolved and total spin moments (in $\mu_{B}$ ) in $\operatorname{PdMnZ}$ and $\mathrm{CuMn} Z$ as a function of the $s p$-electron number of the $Z$ constituent. (b) The same for fullHeusler alloys $\operatorname{Pd}_{2} \mathrm{MnZ}$ and $\mathrm{Cu}_{2} \mathrm{MnZ}$.

of the wave functions in $C 1_{b}$-type crystal structure of the semi-Heusler alloys. ${ }^{4}$ In the following sections, we will show that the position of the occupied Mn $3 d$ states with respect to the Fermi level, the $s p$-electron DOS at the Fermi energy, and the structure of the unoccupied part of the DOS are important for the understanding of the exchange coupling mechanisms in Heusler alloys.

\section{B. Magnetic moments and local moment behavior}

Most of the Mn-based systems are believed to possess well-defined local atomic Mn moments which are usually close to $4 \mu_{B}$ and do not change substantially when going from the ordered phase to the paramagnetic state. The available inelastic neutron scattering experiments and magnetization measurements support this point of view. Early studies of the paramagnetic phase of several Mn-based compounds have established the absence of spatial magnetic correlations (spin waves) and have shown the value of the atomic moment to be in agreement with the moment obtained from the static susceptibility measurements. ${ }^{31}$ Recently, Plogmann et al. gave a detailed study on the degree of magnetic moment localization in various Mn-based full-Heusler alloys using $\mathrm{X}$-ray photoelectron spectroscopy and X-ray emission spectroscopy (XES) techniques. ${ }^{32}$ Depending on the atomic number of the $Z$ element, the authors obtained from XES experiments an increasing localization of the Mn $3 d$ states that is related to the larger interatomic distances for heavier $s p(Z)$ elements. Furthermore, the authors compared the position of the Mn $3 d$ peaks with first- principles calculations and obtained a very good agreement between the experiment and theory. It should, however, be noted that the situation is different in Mn-based intermetallic compounds with smaller Mn-Mn distance (for instance, in systems with NiAs type of the crystal structure) where the first-principles calculations within LSDA or GGA usually underestimate the spin splitting of the Mn $3 d$ states compared to that observed by photoemission and inverse-photoemission spectroscopies. ${ }^{77-79}$

On the theoretical side, in 1984, Kübler et al. gave a detailed account of the formation of local moments in various Mn-based full-Heusler alloys using first-principles calculations. ${ }^{46}$ Since then, many authors have studied various semi- and full-Heusler compounds and came to a similar conclusion. An interesting feature arising from the calculations of Heusler alloys is the resemblance of a number of physical characteristics such as the position and the width of the Mn $3 d$ peaks, exchange splitting, and the value of the Mn magnetic moment to the corresponding quantities for diluted Mn impurities in nonmagnetic metals. ${ }^{80}$ This observation gives further evidence for the localized nature of the magnetism moments in these systems.

In Fig. 3, we present calculated atom-resolved and total magnetic moments for both families of Heusler alloys $\operatorname{PdMnZ}, \mathrm{CuMn} Z, \mathrm{Pd}_{2} \mathrm{MnZ}$, and $\mathrm{Cu}_{2} \mathrm{MnZ}$ as a function of the $s p$-electron number of the $Z$ constituent. As mentioned above, the magnetic moment is mostly confined to the Mn sublattice. Small moments are induced on the $\mathrm{Cu}$ and $\mathrm{Pd}$ atoms. These induced moments are positive (that is, parallel to the Mn moments) in a broad composition interval. The induced moment of the $Z$ element is negative. The induced moments as a function of the $Z$ constituent follow closely the behavior of the Mn moment. This correlation is especially well pronounced for semi-Heusler alloys (Fig. 3). The comparison of the data for $\mathrm{PdMnZ}$ and $\mathrm{CuMnZ}$ shows that for equal total number of valence electrons, the induced moments have similar values. In both semi-Heusler systems, the variation of the total magnetic moment with $Z$ constituent is large. In $\operatorname{PdMn} Z$, it is about $1 \mu_{B}$. The half of the variation comes from the change in the Mn moment. In full-Heusler alloys, the situation is different. Neither a correlation of the characteristics of the compounds with the same number of the valance electrons nor a substantial change in magnetic moments with variation of $Z$ is obtained.

Since an increase of the temperature leads to increasing deviation of the atomic moments from the direction of the net magnetization, it is important to study the properties of the noncollinear magnetic configurations. In Fig. 4, we present the results of such calculations for four stoichiometric compounds: PdMnSn, CuMnSn, $\mathrm{Pd}_{2} \mathrm{MnSn}$, and $\mathrm{Cu}_{2} \mathrm{MnSn}$. The results are shown for the spiral structures with two different wave vectors $\mathbf{q}=\left(00 \frac{1}{2}\right)$ and $\mathbf{q}=(001)$ in units of $2 \pi / a$ and the polar vector $\theta$ varying in the interval from 0 to $90^{\circ}$ [see Eq. (1)].

The upper panels of Fig. 4 show the $\theta$ dependence of the Mn moment, whereas the bottom panels present the $\theta$ dependence of the total energy. Note that by the variation of the wave vector of the spiral and of angle $\theta$, the magnetic struc- 

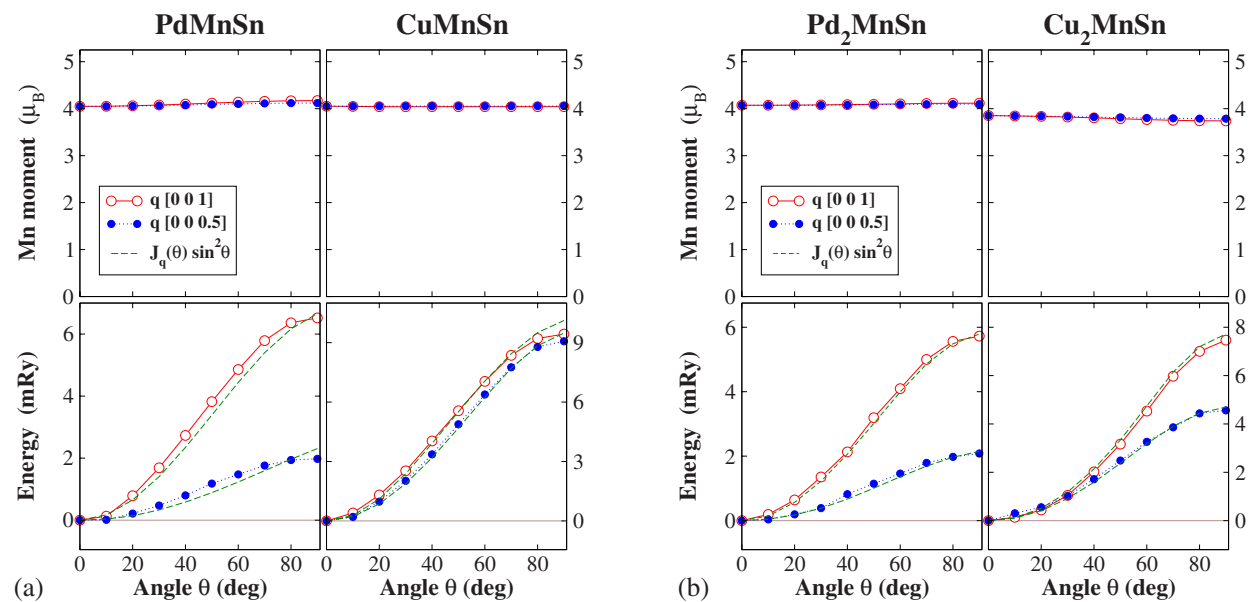

FIG. 4. (Color online) (a): Calculated Mn spin moment in Pd$\mathrm{MnSn}$ and $\mathrm{CuMnSn}$ as a function of $\theta$ for spin spiral with $\mathbf{q}$ $=\left(00 \frac{1}{2}\right),(001)$ in units of $2 \pi / a$. Lower panel: The corresponding total energies $\Delta E(\theta, \mathbf{q})=E(\theta, \mathbf{q})$ $-E(0,0)$. For comparison, the results of the force theorem calculations (broken lines) are presented. $J_{\mathbf{q}}(\theta)$ stand for $J_{\mathbf{q}} \times M_{\theta}^{2}$; where $M$ is the magnetic moment. (b) The same for $\mathrm{Pd}_{2} \mathrm{MnSn}$ and $\mathrm{Cu}_{2} \mathrm{MnSn}$ full-Heusler compounds.

ture can be continuously transformed from ferromagnetic to antiferromagnetic. The spirals with $\theta=0$ and arbitrary $\mathbf{q}$ correspond to the ferromagnetic state, whereas the structure with $\theta=90^{\circ}$ and $\mathbf{q}=(001)$ is antiferromagnetic.

The analysis of Fig. 4 shows that for all four compounds, the magnetic moment of the Mn atom is practically insensitive to the magnetic configuration. The relative change of the Mn moment in transition from ferromagnetic to antiferromagnetic state is less than $3 \%$. Therefore, the treatment of the Mn moment in these systems as a local property of the $\mathrm{Mn}$ atom is well founded.

The energy of the spiral structures increases monotonously with increasing $\theta$ and reaches the maximal value for the antiferromagnetic state. The minimum at $\theta=0$ shows that the ground state is ferromagnetic. In Fig. 4, we compare the total energy calculated self-consistently with an approximation to the total energy obtained with the application of the so-called magnetic force theorem. ${ }^{81}$ The agreement between two types of calculations is good in the whole $\theta$ interval that is characteristic for systems with well-defined atomic moments and allows the use of the force theorem for magnetic configurations deviating strongly from the ground state.

\section{EXCHANGE COUPLING MECHANISM}

In this section, we suggest an interpretation of the results of the first-principles calculations on the basis of the Anderson $s$ - $d$ mixing model. The discussion is divided into four parts. In the first part, the calculated Heisenberg exchange parameters are presented. In the second part, we briefly describe the Anderson $s$ - $d$ mixing model and the exchange mechanisms resulting from the perturbative treatment of this model. The role of the conduction electron spin polarization in exchange coupling as well as the contribution of the $s p$ and nonmagnetic $3 d$ atoms to the formation of the magnetic state is discussed in the third part. The last part is devoted to the study of the influence of nonmagnetic $3 d$ versus $4 d$ atoms on the exchange coupling.

\section{A. Heisenberg exchange parameters}

In Fig. 5, we present calculated Mn-Mn exchange parameters for six nearest neighbors as a function of $s p$-electron concentration. In the upper-right corner of each panel, the number of atoms in the corresponding coordination sphere is given. The exchange parameters for larger interatomic dis-

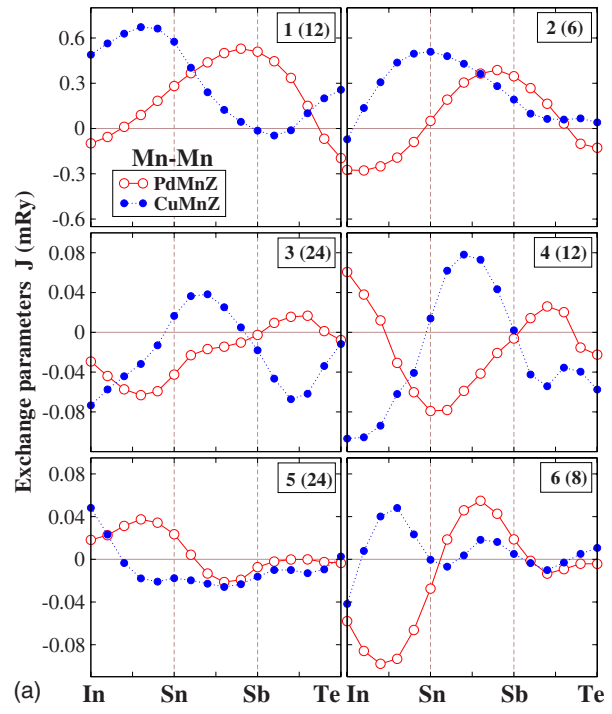

FIG. 5. (Color online) (a): First six nearest neighbor Mn-Mn exchange parameters in $\mathrm{PdMnZ}$ and $\mathrm{CuMn} Z$ as a function of the sp-electron number of the $Z$ constituent. Also given are the number of atoms within corresponding coordination spheres. (b): The same for full-Heusler alloys $\mathrm{Pd}_{2} \mathrm{MnZ}$ and $\mathrm{Cu}_{2} \mathrm{MnZ}$. 


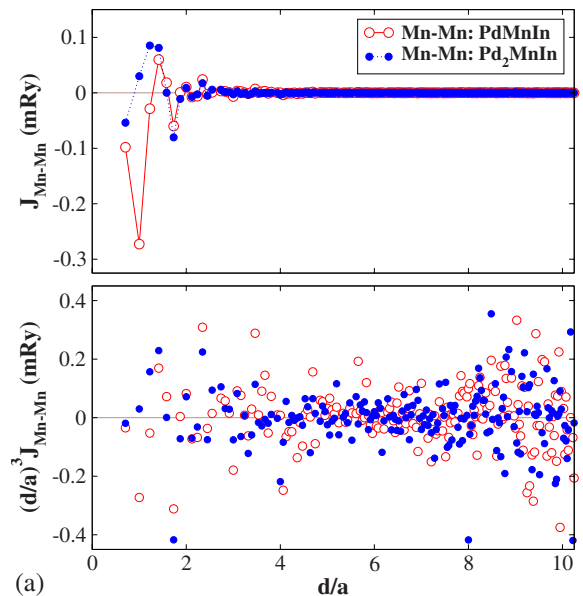

(a)

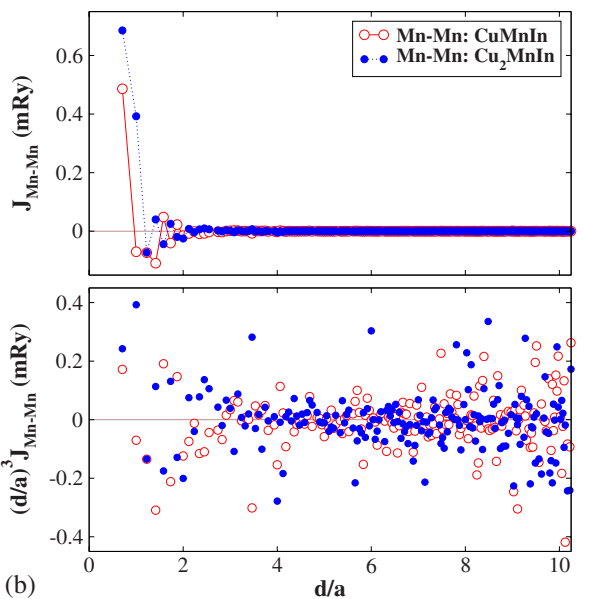

FIG. 6. (Color online) (a): Mn-Mn exchange interactions in $\operatorname{Pd}_{k} \operatorname{MnIn}(k=1,2)$ as a function of distance up to the $10 a$. Lower panel: RKKY-type oscillations in exchange parameters for corresponding compounds. (b) The same for $\mathrm{Cu}_{k} \mathrm{MnIn}(k=1,2)$ tances $(\sim 10 a)$ are shown in Fig. 6 for selected compounds PdMnIn, CuMnIn, $\mathrm{Pd}_{2} \mathrm{MnIn}$, and $\mathrm{Cu}_{2} \mathrm{MnIn}$. The absolute value of the exchange parameters decays quickly with increasing interatomic distance and the main contribution to $T_{C}$ comes from the interaction between atoms lying closer than $3 a$. No sizable contribution is detected after $5 a$. However, the RKKY-type oscillations become visible up to very large interatomic distances when the exchange parameters are multiplied by $(d / a)^{3}$ (Fig. 6), where $d$ is the distance between the interacting $\mathrm{Mn}$ atoms and $a$ is the lattice constant.

In agreement with the results of our previous calculations on Heusler alloys, a strong dependence of the exchange parameters on the $Z$ constituent for both families of systems is obtained. As seen from Fig. 5, all exchange parameters oscillate between ferromagnetic and antiferromagnetic values with increasing $s p$-electron concentration. As discussed below, these oscillations are related to the properties of the electron structure of the systems.

Considering two nearest neighbor exchange parameters, we notice that they have a ferromagnetic character for a broad composition range and dominate over the rest of parameters. The remaining parameters are much weaker. The first two nearest neighbor exchange interactions are responsible for very high Curie temperatures in $\mathrm{Cu}$-based fullHeusler alloys as well as in both classes of semi-Heusler alloys.

A distinct feature of the exchange interactions in semiHeusler alloys is that the maximum of the exchange interactions for both $\mathrm{PdMnZ}$ and $\mathrm{CuMnZ}$ corresponds to the similar numbers of the $s p$ electrons. This correlates with the conclusions of the preceding sections where a similar behavior was obtained for the density of states and the magnetic moments. The shift of the maxima for two systems is explained by the fact that $\mathrm{Pd}$ has one $s p$ electron less than $\mathrm{Cu}$. The properties of the exchange interactions are reflected in the properties of the Curie temperature (Fig. 7) where we also obtained a relative shift of the maxima of the two curves corresponding to one $s p$ electron. However, no such correlation is obtained for the full-Heusler compounds.

\section{B. Indirect exchange coupling: Ruderman-Kittel-Kasuya- Yoshida-type exchange and superexchange}

In previous calculations on $\mathrm{Co}_{2} \mathrm{MnSi}$ and $\mathrm{Mn}_{2} \mathrm{VAl}$, we have shown that in the systems with several magnetic sub-
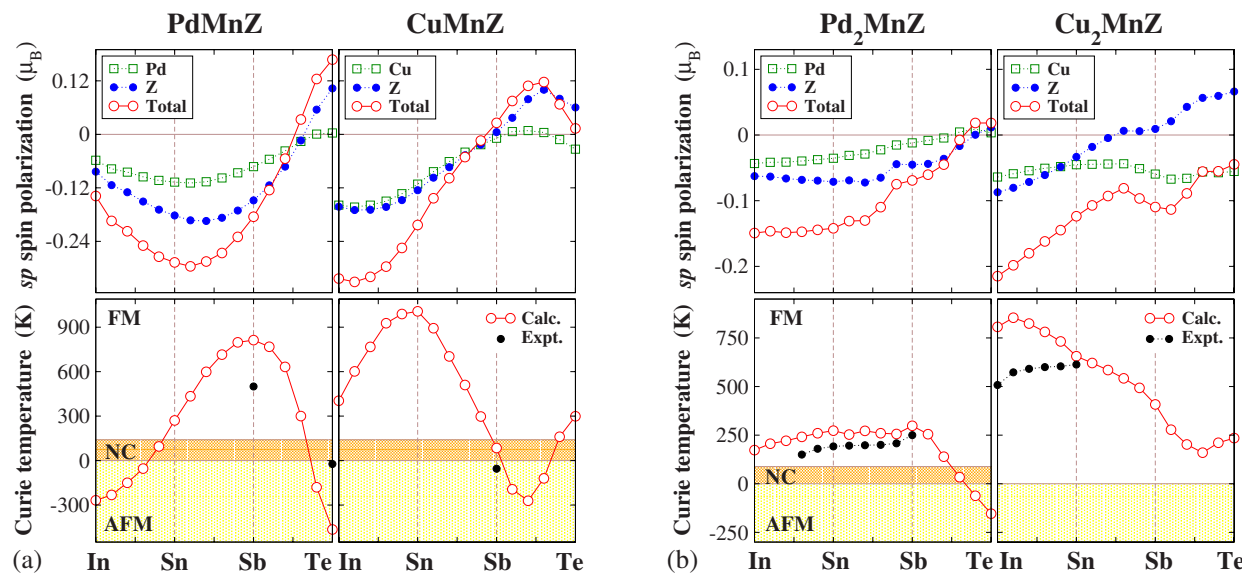

FIG. 7. (Color online) (a): The $s p$-electron spin polarization (in $\mu_{B}$ ) of the $X=\mathrm{Pd}, \mathrm{Cu}$ and $Z$ constituents. The total polarization is given as the sum of $X$ and $Z$ spin polarizations. Lower panel: Mean-field estimation of the Curie temperature in PdMnZ and CuMnZ. For comparison, available experimental $T_{C}$ values taken from Refs. 1 and 2 are presented. FM, NC, and AFM stand for ferromagnetic, noncollinear, and antiferromagnetic ordering, respectively. (b) The same for the full-Heusler alloys $\operatorname{Pd}_{2} \mathrm{MnZ}$ and $\mathrm{Cu}_{2} \mathrm{MnZ}$. 
lattices, the direct exchange coupling $\mathrm{Mn}-\mathrm{Co}$ or $\mathrm{Mn}-\mathrm{V}$ between neighboring $3 d$ atoms can dominate over the indirect Mn-Mn interactions. ${ }^{49}$ For the Mn-based Heusler compounds considered in this paper, the direct coupling does not play a substantial role and can be ignored. ${ }^{47}$ Therefore, in the following, we will consider only the indirect exchange coupling.

The DFT is not based on a model Hamiltonian approach and does not use a perturbative treatment. Therefore, various exchange mechanisms appear in the results of calculations in a mixed form that does not allow a straightforward separation of the contributions of different mechanisms. In this situation, the model Hamiltonian studies relevant to the problem provide useful information for a qualitative interpretation of the DFT results. Among such approaches, the Anderson $s$ - $d$ mixing model is an appropriate tool for Mn-based Heusler alloys because of the localized nature of Mn moments in these systems.

Anderson $s$ - $d$ model for a single $3 d$ impurity embedded into a nonmagnetic metallic host is given by the following Hamiltonian: ${ }^{40}$

$$
\begin{aligned}
H_{s-d}= & \sum_{\mathbf{k} \sigma} \epsilon_{\mathbf{k}} a_{\mathbf{k} \sigma}^{\dagger} a_{\mathbf{k} \sigma}+\sum_{\sigma} \epsilon_{d} n_{d \sigma}+\sum_{\mathbf{k} \sigma} V_{\mathbf{k} d}\left(a_{\mathbf{k} \sigma}^{\dagger} a_{d \sigma}+a_{\mathbf{k} \sigma} a_{d \sigma}^{\dagger}\right) \\
& +U n_{d \uparrow} n_{d \downarrow},
\end{aligned}
$$

where $a_{\mathbf{k} \sigma}^{\dagger}\left(a_{\mathbf{k} \sigma}\right)$ and $a_{d \sigma}^{\dagger}\left(a_{d \sigma}\right)$ create (annihilate) electrons with spin $\sigma$ in the band states and on the impurity, respectively, $n_{d \sigma}$ is the number operator for localized electrons of spin $\sigma$, and $U$ is the on-site Coulomb repulsion between two localized electrons which favors the single occupation of the impurity level. $V_{\mathbf{k} d}$ represents the coupling between the impurity level and the conduction electrons in the metal. This interaction causes the mixing of the band states and impurity level. Such a mixing gives rise to broadening of the impurity states. The ratio $U / V$ determines whether a local magnetic moment in a metallic host can be formed. The first-principles calculations for $3 d$ impurities in nonmagnetic metals gave large values for the magnetic moments reflecting the strong Coulomb repulsion $U$ between $3 d$ orbitals. ${ }^{80}$ Note that in complex systems such as rare earths and Heusler alloys, one deals with a lattice of magnetic atoms. In this case, the relevant model is the periodic Anderson $s-d$ model. ${ }^{82-85}$

The mixing interaction $V$ induces spin polarization in the conduction electron sea and the spatial propagation of this polarization gives rise to an effective indirect exchange coupling between distant magnetic moments. In general, this interaction contains two distinct processes: electrostatic Coulomb exchange interaction and $s-d$ (or $s p-d$ ) mixing interaction. The former induces a net positive spin polarization, while the contribution of the latter is negative and disappears in the strong magnetic limit. ${ }^{86}$ The indirect exchange coupling has been described using various theoretical schemes. The Green's function method and the perturbation theory are among the most often used techniques. In 1973, Gonçalves Da Silva and Falicov showed that, in fourth-order perturbation theory, the indirect interaction between two magnetic moments can be separated into two contributions: ${ }^{62} J_{\text {indirect }}$ $=J_{R K K Y}+J_{S}$. The first term is of RKKY type and stems from the intermediate states which correspond to low-energy spin excitations of the Fermi sea, that is, excitations corresponding to the electron-hole pair formation with a spin flip transition. In reciprocal space, this contribution takes the following form: ${ }^{62,64-66}$

$$
\begin{aligned}
J_{R K K Y}(\mathbf{q})= & \sum_{n_{1}, n_{2}, k}\left[\frac{\left.\left|V_{n_{1}} k^{2}\right| V_{n_{2} k^{\prime}}\right|^{2}}{\left(\varepsilon_{n_{2} k^{\prime}}-\varepsilon_{d}\right)^{2}} \frac{\theta\left(\varepsilon_{f}-\varepsilon_{n_{1}}\right) \theta\left(\varepsilon_{n_{2} k^{\prime}}-\varepsilon_{f}\right)}{\varepsilon_{n_{2} k^{\prime}}-\varepsilon_{n_{1} k}}\right. \\
& + \text { c.c. }],
\end{aligned}
$$

where $\mathbf{k}^{\prime}=\mathbf{k}+\mathbf{q}+\mathbf{G}$ and $\mathbf{G}$ is the reciprocal lattice vector. This coupling is largely influenced by the denominator $\left(\varepsilon_{n_{2} k^{\prime}}-\varepsilon_{n_{1} k}\right)$. Therefore, the topology of the Fermi surface is an important factor influencing the form of $J_{R K K Y}(\mathbf{q})$ with most important contributions coming from the stationary wave vectors spanning the Fermi surface. These stationary wave vectors translate into smoothly decaying oscillations in the real space with a ferromagnetic bias in the "preasymptotic" regime. Also, the DOS at the Fermi level, the number of conduction electrons, and their spin polarization play an important role in determining the strength of this interaction.

The second term arises from high-energy virtual charge excitations in which electrons from local $3 d$ states of the magnetic atom are promoted above the Fermi sea. In the reciprocal space, the term has the following form that is similar to the form of the RKKY-type term:

$$
\begin{aligned}
J_{S}(\mathbf{q})= & -\sum_{n_{1}, n_{2}, k}\left[\frac{\left|V_{n_{1} k}\right|^{2}\left|V_{n_{2} k^{\prime}}\right|^{2}}{\left(\varepsilon_{n_{2} k^{k^{\prime}}}-\varepsilon_{d}\right)^{2}} \frac{\theta\left(\varepsilon_{n_{1} k}-\varepsilon_{f}\right) \theta\left(\varepsilon_{n_{2} k^{\prime}}-\varepsilon_{f}\right)}{\varepsilon_{n_{1} k}-\varepsilon_{d}}\right. \\
& + \text { c.c. }] .
\end{aligned}
$$

In contrast to the RKKY contribution, $J_{S}(\mathbf{q})$ does not, however, depend on the DOS at the Fermi level and the topology of the Fermi surface. Since the sum is taken over unoccupied states, an important role is played by the energy position of the unoccupied $3 d$ states of the magnetic atom. The closer the states to the Fermi level, the larger $J_{S}(\mathbf{q})$. This interaction is always antiferromagnetic and its strength decays exponentially with distance. A broadening of the $3 d$ levels induces weak oscillations in this coupling. In addition to these parameters, the position of the occupied $3 d$ levels with respect to the Fermi energy and the strength of the mixing interaction $V$ strongly influence coupling mechanisms.

As shown in Ref. 66, the $\mathbf{q} \rightarrow 0$ limit simplifies the above expressions and is useful for the qualitative analysis. For $\mathbf{q}$ $=0$, the RKKY-type term becomes

$$
J_{R K K Y}(0)=V^{4} D\left(\epsilon_{F}\right) / E_{h}^{2},
$$

where $D\left(\epsilon_{F}\right)$ is the density of states at the Fermi level and $E_{h}$ is the energy required to promote an electron from the occupied $3 d$ level to the Fermi level. Parameter $E_{h}$ is not well defined in Heusler alloys because of the broadening of the Mn $3 d$ levels into the energy bands crossing the Fermi level. In all alloys studied, the occupied Mn $3 d$ peaks lie below -0.1 Ry where the energy is counted off the Fermi level 
(Fig. 2). On the other hand, the $\mathbf{q}=0$ limit of the superexchange term cannot be expressed in terms of the density of states and has more complex form

$$
J_{S}(0)=V^{4} \sum_{n k}\left[\epsilon_{F}-\epsilon_{n k}-E_{h}\right]^{-3} .
$$

In addition, we would like to comment on the following two points. First, although the perturbative derivation of above expressions is based on the assumption of two magnetic impurities embedded into the metallic host, the generalization to the periodic lattices is straightforward. As shown by Malmström et al. ${ }^{43}$ and Price, ${ }^{44}$ the final results differ by a phase factor. ${ }^{43,44}$ Second, there are two different limits in the description of the exchange mechanisms within the Anderson $s-d$ model. In the weak magnetic limit where the coupling is dominated by the $s-d$ mixing of the local and conduction electron states, both mechanisms mentioned above coexist and their relative contributions are determined by the details of the electronic structure of the system. In the opposite limit (strong magnetic limit) in which the coupling is primarily due to the electrostatic Coulomb exchange interaction, the second term is not present and the first term is reduced to the conventional RKKY interaction. ${ }^{66}$ The systems considered in this paper are characterized by the strong $s$ - $d$ hybridization and therefore we expect the presence of the contributions of both exchange mechanisms.

\section{Conduction electron spin polarization}

Now, we turn to the interpretation of the DFT results in terms of the two exchange mechanisms discussed in the previous section. To estimate the relative contribution of the ferromagnetic RKKY-type exchange, we present in Fig. 7 the calculated conduction electron spin polarization in both families of Heusler alloys as a function of the electron number of the $Z$ constituent. The analysis of the calculation data allows us to draw a number of important conclusions. First, in both families of alloys, the direction of the induced spin polarization is opposite to the direction of the Mn moment in a broad interval of compositions. This feature reveals the primary role of the $s p-d$ mixing in the exchange coupling and justifies the use of the Anderson $s$ - $d$ model for the description of the magnetism in these systems.

Another remarkable feature is a very clear correlation between the spin polarization and the mean-field Curie temperature (or exchange parameters) in a large part of the phase diagram (Fig. 7). Indeed, the compounds with a very large spin polarization are characterized by the value of the Curie temperature that is also very high. Interestingly, for the zero polarization, the Curie temperature also vanishes or assumes very small values reflecting the dominating character of the ferromagnetic RKKY-type exchange mechanism in establishing magnetic order. However, at some regions, superexchange mechanism becomes important. This can be seen in Fig. 7 where for the $\mathrm{PdMnIn}_{1-x} \mathrm{Sn}_{x}(x<0.8)$ system, we obtain an antiferromagnetic order in spite of a very large spin polarization. Further insight into the nature of the coupling can be gained from Fig. 2 where we present atom and spin resolved densities of states of both families of compounds for stoichiometric compositions. As pointed out above, the superexchange mechanism is sensitive to the DOS above the Fermi energy. As seen in Fig. 2, the Mn $3 d$ states provide the main contribution to the DOS in this region. For PdMnIn, the DOS peak above the Fermi level assumes the largest value and, as a result, the antiferromagnetic superexchange dominates over the ferromagnetic RKKY-type exchange, giving rise to antiferromagnetic (AFM) order. In transition from In to $\mathrm{Sn}$, the peak gradually decreases and therefore the superexchange becomes less important. Around $Z=S b$, it almost disappears, leading to ferromagnetic (FM) order. However, another large peak approaches the Fermi level for $Z=T e$ that turns the systems from a ferromagnet into an antiferromagnet with a noncollinear ordering in the intermediate region due to the competition of two mechanisms. The situation is very similar in the case of $\mathrm{CuMnZ}$ where we also obtain a rich magnetic behavior.

On the other hand, for the full-Heusler alloys, the magnetic phase diagram is rather simple compared to the semiHeusler systems. In $\mathrm{Cu}_{2} \mathrm{MnZ}$, the ground state is ferromagnetic for all $Z$. The mean-field $T_{C}$ qualitatively follows the behavior of the conduction electron spin polarization, assuming the largest value for $Z=\operatorname{In}$ and gradually decreasing in the In-Sn-Sb-Te sequence. Around Sb, the conduction electron spin polarization increases that, in principle, should lead to the increase of the Curie temperature according to the discussion of the previous section. However, in this region, the $T_{C}$ further decreases, revealing substantial contribution of the antiferromagnetic superexchange. This is reflected in position of the Mn $3 d$ states that is very close to the Fermi level (Fig. 2).

In the Pd-based full-Heusler alloys $\mathrm{Pd}_{2} \mathrm{MnZ}$, the spin polarization is rather small and, as a result, the Curie temperature is low. The spin polarization in In-Sn interval is independent of the $Z$ constituent, while in the rest of the phase diagram, it gradually decreases and becomes zero around Te. As for the $T_{C}$, we obtain a similar $Z$-independent behavior in a large interval of compositions (from In to Sb). However, from $\mathrm{Sb}$ to $\mathrm{Te}$, the $T_{C}$ sharply decreases due to the dominating contribution of superexchange. The calculated $T_{C}$ values are in good agreement with the experiments for the Pd-based full Heusler alloys, while they are overestimated in $\mathrm{PdMnSb}$ and $\mathrm{Cu}$-based full-Heusler compounds. The following explanation of this property can be suggested. In the Pd-based full-Heusler alloys, the more distant exchange parameters contribute with a substantial weight to the formation of the Curie temperature (Fig. 5), whereas in $\mathrm{PdMnSb}$ and $\mathrm{Cu}-$ based full-Heusler compounds, only the first and second nearest neighbor parameters determine the $T_{C}$. In the latter case, the mean-field approximation is less exact and overestimates the Curie temperature. For such systems, the random-phase approximation is expected to provide a better description of the $T_{C}$.

The results of the first-principles calculations and the qualitative analysis on the basis of the Anderson $s$ - $d$ model allow us to formulate two conditions for a high Curie temperature. (i) The conduction electron spin polarization should be maximal. (ii) The DOS above the Fermi level should be minimal. Indeed, as seen from Figs. 2 and 7, the compounds $\mathrm{CuMnSn}, \mathrm{PdMnSb}$, and $\mathrm{Cu}_{2} \mathrm{MnIn}$ that satisfy both condi- 

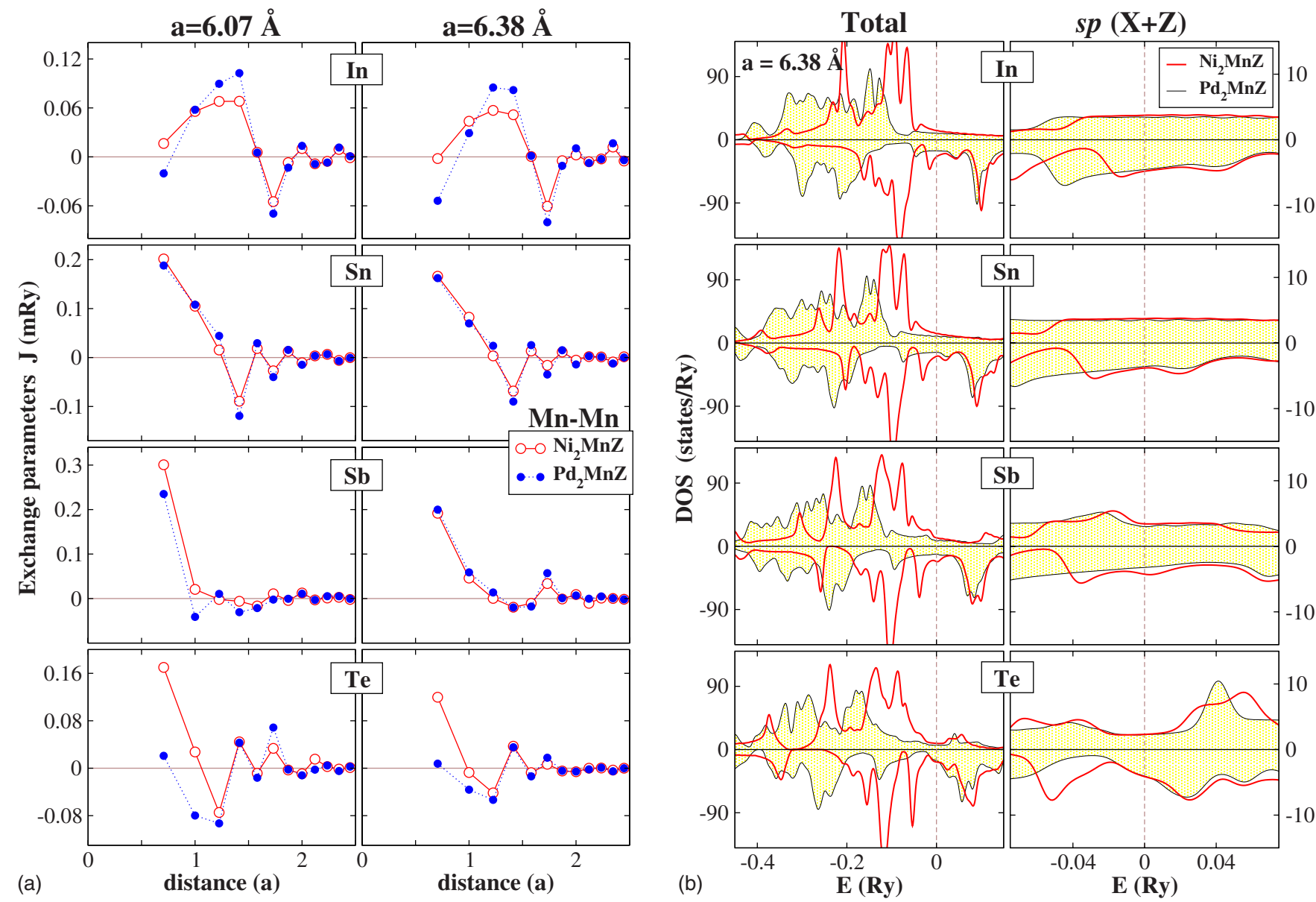

FIG. 8. (Color online) (a): The parameters of the Mn-Mn exchange interactions in $\mathrm{Ni}_{2} \mathrm{MnZ}$ and $\mathrm{Pd}_{2} \mathrm{Mn} Z(Z=\mathrm{In}, \mathrm{Sn}, \mathrm{Sb}, \mathrm{Te})$ for two different lattice constants. (b): Spin projected total DOS (left hand side) and $s p$-electron DOS of $X$ and $Z$ atoms (right hand side) for Ni ${ }_{2} \mathrm{MnZ}$ and $\mathrm{Pd}_{2} \mathrm{MnZ}(\mathrm{Z}=\mathrm{In}, \mathrm{Sn}, \mathrm{Sb}, \mathrm{Te})$ for the lattice constants of $6.38 \AA$.

tions possess very high Curie temperatures. The knowledge of the conditions for a high Curie temperature is an important help in the fabrication of the materials with desired properties. From the point of view of the second condition, the half-metallic ferromagnets have an advantage since the gap in the spin-down channel decreases the number of the states just above the Fermi level. Indeed, a large number of first-principles calculations showed that these materials have very high Curie temperatures. ${ }^{87-93}$

As mentioned in preceding part, the direction of the conduction electron spin polarization with respect to the local moment and its amplitude are important in classifying ferromagnets. From the obtained results, the full-Heusler alloys can be characterized as weak ferromagnets since the spin polarization is negative for all $Z$ constituents, i.e., $s p-d$ mixing is dominating. As for the semi-Heusler alloys, the situation is similar to the full-Heusler alloys except some regions in the magnetic phase diagram where the spin polarization changes sign: $\mathrm{Sb}-\mathrm{Te}$ interval in $\mathrm{CuMnSb}$ and the region around Te in $\operatorname{PdMnZ}$. A positive spin polarization does not directly mean that these alloys are strongly ferromagnetic. They are, however, close to this limit and the Mn-Mn coupling is primarily due to the electrostatic Coulomb exchange interaction. Indeed, as seen from Figs. 3 and 7, the alloys with large positive spin polarization have large Mn magnetic moments. In PdMnTe, both quantities assume the largest values: $m_{\mathrm{Mn}}=4.4 \mu_{B}$ and $m_{\mathrm{sp}}=0.18 \mu_{B}$. The dependences of the Mn moment and of the spin polarization on the $Z$ constituent are correlated (Figs. 3 and 7).

Because of the importance of the conduction electron spin polarization, a direct comparison with the experimental measurement of this quantity is desirable. An important information on the conduction electron spin polarization is supplied by the measurements of the hyperfine fields at nonmagnetic sites $(X, Z)$. The strength of the transferred hyperfine fields correlates with the amplitude of the $s$ conduction electron spin polarization. Indeed, the measurements by Campbell ${ }^{94}$ and Khoi et al. ${ }^{95}$ showed that maximal $s$ electron spin polarization is found in the systems with high Curie temperatures such as $\mathrm{Cu}_{2} \mathrm{MnAl}$ and $\mathrm{Cu}_{2} \mathrm{MnIn}$. However, the hyperfine fields are sensitive to only the $s$ electron spin polarization and do not give information about the polarization of the $p$ electrons that are usually dominating in Heusler alloys. For probing the total conduction electron spin polarization, the magnetic Compton scattering profiles are proved to be a useful tool. Using this method, Zukowski et al. obtained recently a large $s p$-electron spin polarization in $\mathrm{Cu}_{2} \mathrm{MnAl}$ which is antiferromagnetically coupled to the $\mathrm{Mn}$ moments. ${ }^{96}$ A similar result is obtained by Deb et al. for 
$\mathrm{Ni}_{2} \mathrm{MnSn} .{ }^{97}$ Our calculations are in agreement with both experiments.

\section{D. $3 d$ versus $4 d$ electrons}

In the majority of the Heusler alloys with chemical formulas $X_{2} Y Z$ and $X Y Z$, the $Y$ site is occupied by the Mn atom, while for the $X$ site, there is much more freedom: here, can be any element from $3 d, 4 d$, or $5 d$ late transition metals (i.e., $\mathrm{Fe}, \mathrm{Ru}, \mathrm{Co}, \mathrm{Rh}, \mathrm{Ni}, \mathrm{Pd}, \mathrm{Pt}, \mathrm{Cu}, \mathrm{Ag}$, and $\mathrm{Au}$ ). The magnetic moment of the $X$ atom can, for many alloys, be neglected. The exceptions are some of the $3 d$ atoms, e.g., Fe or Co. Since the delocalization of the $d$ states increases with transition from $3 d$ elements to $4 d$ and further to $5 d$ elements, one can expect a substantial dependence of the magnetic properties of the Heusler alloys for the occupation of the $X$ site with atoms from different $d$ series. The purpose of this section is to study this dependence by means of comparison of two systems: $\mathrm{Ni}_{2} \mathrm{MnZ}$ and $\mathrm{Pd}_{2} \mathrm{MnZ}(Z=\mathrm{In}, \mathrm{Sn}, \mathrm{Sb}, \mathrm{Te})$.

We choose two different lattice parameters for each system: 6.07 and $6.38 \AA$. The first corresponds to the lattice constant of Ni-based alloys, while the second is characteristic of the Pd-based systems. Both systems are experimentally well studied. In Fig. 8, we present the spin projected total DOS and $s p$-electron DOS of the $X$ and $Z$ atoms for the lattice constant of $6.38 \AA$. Note that $\mathrm{Ni}(3 d)$ is isoelectronic to $\mathrm{Pd}(4 d)$. The calculated magnetic moments are given in Table II. The comparison of the results obtained for the same lattice constant reveals similarity of some features: the value of the Mn magnetic moment, $s p$-electron spin polarization, and DOS of the $s p$ states of $X$ and $Z$ atoms around the Fermi level (see Fig. 8 and Table II). On the other hand, well below the Fermi energy, the DOS of the Ni-based and Pd-based systems differ strongly. The peaks of the DOS of the Nibased compounds are higher and narrower than in the Pdbased systems. This is mainly the result of a more delocalized character of the Pd $4 d$ orbitals compared to the Ni $3 d$ orbitals. As we will show, despite the strong difference of the occupied part of the DOS, the magnetic properties of two systems are rather close that give additional support to the conclusion that the states lying close to the Fermi level play the most important role in the formation of magnetic properties.

In Fig. 8 (left panel), we present the parameters of the Mn-Mn exchange interactions for $\mathrm{Ni}_{2} \mathrm{MnZ}$ and $\mathrm{Pd}_{2} \mathrm{MnZ}(Z$ $=\mathrm{In}, \mathrm{Sn}, \mathrm{Sb}, \mathrm{Te}$ ) for two different values of the lattice constant. The Curie temperatures estimated within the meanfield approximation are given in Table II. For both systems, the patterns of exchange parameters for the equal lattice constants are rather similar. The parameters are very close to each other for $Z=S n$ and $\mathrm{Sb}$. For $Z=\mathrm{Te}$, the difference is stronger. This difference is more pronounced for near neighbors. For example, the difference in the first two exchange parameters makes $\mathrm{Pd}_{2} \mathrm{MnTe}$ antiferromagnetic in contrast to the ferromagnetic $\mathrm{Ni}_{2} \mathrm{MnTe}$.

The analysis in terms of the competition between two types of exchange interactions suggested in the previous section is helpful also in this case. The long range behavior of the exchange parameters, specifically the RKKY-type oscillations, can be related to the $s p$-electron spin polarization and total $s p$-electron DOS at the Fermi level. As seen from Table II and Fig. 8, these quantities are very close to each other in both systems that explains the similarity in the long range behavior of the exchange interactions. However, the short range behavior depends very much on the contribution of the antiferromagnetic superexchange. To reveal the importance of this mechanism, we present in Fig. 9 the total DOS (spin up plus spin down) above the Fermi energy for both systems and for the lattice constant of $6.38 \AA$. As seen in Fig. 9, the DOS above the Fermi level is not identical for $X=\mathrm{Ni}$ and $X=\mathrm{Pd}$. Therefore, the deviations in exchange parameters at least within a few coordination spheres can be expected. A very strong similarity in the patterns of the exchange parameters for $\mathrm{Sn}$ and $\mathrm{Sb}$ alloys compared to the $\mathrm{In}$ and Te systems cannot be explained on the basis of the differences in the DOS and might be accidental resulting from the cancellation of the contributions of different states to the superexchange.

Next, we comment on the volume dependence of the magnetic properties. As seen in Table II and Fig. 8, the compres-

TABLE II. Magnetic moments, $s p$-electron polarization (in $\left.\mu_{B}\right)$, and Curie temperatures in $\mathrm{Ni}_{2} \mathrm{MnZ}_{\text {and }} \mathrm{Pd}{ }_{2} \mathrm{MnZ}(Z=\mathrm{In}, \mathrm{Sn}, \mathrm{Sb}, \mathrm{Te})$ for two different lattice parameters. Negative values of $T_{C}$ mean that the ground state is antiferromagnetic.

\begin{tabular}{|c|c|c|c|c|c|c|c|c|c|c|c|c|}
\hline \multirow[b]{2}{*}{ Compound } & \multicolumn{6}{|c|}{$a=6.07 \AA$} & \multicolumn{6}{|c|}{$a=6.38 \AA$} \\
\hline & $X$ & $\mathrm{Mn}$ & $Z$ & $s p$ & Cell & $\begin{array}{c}T_{C}^{M F A} \\
(\mathrm{~K})\end{array}$ & $X$ & $\mathrm{Mn}$ & $Z$ & $s p$ & Cell & $\begin{array}{c}T_{C}^{M F A} \\
(\mathrm{~K})\end{array}$ \\
\hline $\mathrm{Ni}_{2} \mathrm{MnIn}$ & 0.27 & 3.72 & -0.07 & -0.16 & 4.19 & 254 & 0.27 & 3.99 & -0.07 & -0.17 & 4.45 & 165 \\
\hline $\mathrm{Pd}_{2} \mathrm{MnIn}$ & 0.11 & 3.81 & -0.05 & -0.14 & 3.98 & 279 & 0.17 & 4.10 & -0.05 & -0.15 & 4.26 & 173 \\
\hline $\mathrm{Ni}_{2} \mathrm{MnSn}$ & 0.21 & 3.74 & -0.06 & -0.13 & 4.10 & 321 & 0.21 & 4.00 & -0.06 & -0.14 & 4.35 & 251 \\
\hline $\mathrm{Pd}_{2} \mathrm{MnSn}$ & 0.09 & 3.79 & -0.06 & -0.13 & 3.91 & 368 & 0.07 & 4.07 & -0.06 & -0.14 & 4.15 & 263 \\
\hline $\mathrm{Ni}_{2} \mathrm{MnSb}$ & 0.15 & 3.76 & -0.03 & -0.09 & 4.04 & 309 & 0.22 & 4.04 & -0.02 & -0.06 & 4.46 & 234 \\
\hline $\mathrm{Pd}_{2} \mathrm{MnSb}$ & 0.08 & 3.82 & -0.03 & -0.08 & 3.95 & 193 & 0.09 & 4.13 & -0.03 & -0.07 & 4.29 & 296 \\
\hline $\mathrm{Ni}_{2} \mathrm{MnTe}$ & 0.22 & 3.83 & 0.03 & 0.01 & 4.31 & 120 & 0.21 & 4.04 & 0.02 & -0.03 & 4.48 & 46 \\
\hline $\mathrm{Pd}_{2} \mathrm{MnTe}$ & 0.14 & 3.89 & 0.04 & 0.04 & 4.21 & -172 & 0.13 & 4.17 & 0.03 & 0.02 & 4.45 & -154 \\
\hline
\end{tabular}




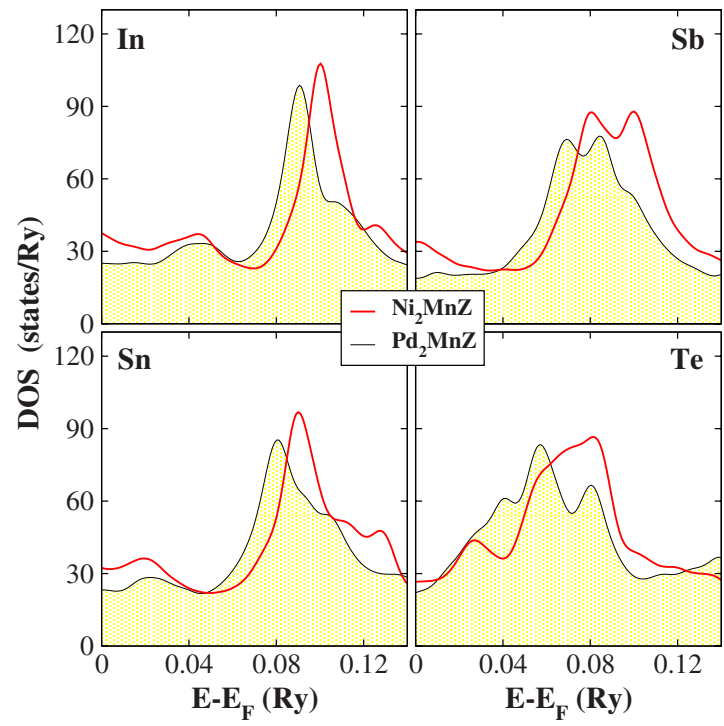

FIG. 9. (Color online) The total DOS of $\mathrm{Ni}_{2} \mathrm{MnZ}$ and $\mathrm{Pd}_{2} \mathrm{MnZ}$ $(Z=\mathrm{In}, \mathrm{Sn}, \mathrm{Sb}, \mathrm{Te})$ above the Fermi level.

sion of the volume (the reduction of the lattice parameter) leads to the decrease of the Mn magnetic moments and an increase of the absolute value of the leading exchange parameters. The reduction of the magnetic moments is an expected result which is a consequence of the increased interatomic hybridization and broadening of the electron bands. The volume dependence of the exchange interactions is less straightforward. In general, this dependence is nonmonotonous, and in concrete physical situations, one can find both an increase and a decrease of the exchange interactions with volume contraction. A detailed discussion of these aspects can be found in Ref. 48 where the electronic structure, exchange interactions, and Curie temperature of the fullHeusler alloy $\mathrm{Ni}_{2} \mathrm{MnSn}$ are studied as a function of pressure. It was shown that in the low pressure region, the $T_{C}$ behavior is in qualitative correlation with the empirical interaction curve of Kanomata et al., which describes the dependence of the Curie temperature of the Mn-based Heusler alloys on the Mn-Mn distance. ${ }^{98}$ In agreement with the experiment, we have found that at ambient pressure, $T_{C}$ increases with increasing pressure, that is, $d T_{C} / d P>0 .{ }^{98-100}$ The pressure dependence has a maximum at $3.6 \AA$. Indeed, as seen in Table II, in agreement with experiments, we obtain the same behavior in the $T_{C}\left(T_{N}\right.$ for $\left.\mathrm{Pd}_{2} \mathrm{MnTe}\right)$ for all compounds except $\mathrm{Pd}_{2} \mathrm{MnSb}$ in which the Curie temperature decreases with the reduction of Mn-Mn distance. To summarize, the magnetism of the Heusler alloys containing different $d(3 d$ or $4 d$ ) electrons appeared to be qualitatively similar, whereas quantitative differences result from the stronger delocalization of the $4 d$ electrons and larger lattice parameters of the compounds having $4 d$ elements.

\section{SUMMARY AND CONCLUSIONS}

We performed a systematic first-principles study to reveal the exchange mechanisms in various Mn-based semi- and full-Heusler alloys. The calculation of the exchange parameters is based on the frozen-magnon approach and the Curie temperature is estimated within the mean-field approximation. Due to large separation of the Mn atoms and the local moment nature of magnetism in these systems, the exchange coupling is indirect and is mediated by the conduction electrons. The results obtained are interpreted using the $s-d$ mixing model of Anderson. To understand the dependence of physical characteristics on the valence electron number, we go beyond the stoichiometric compositions employing the virtual crystal approximation. The influence of nonmagnetic $3 d$ versus $4 d$ electrons on exchange coupling is discussed.

We show that magnetism in these systems strongly depends on the number of conduction electrons, their spin polarization, and the position of the unoccupied Mn $3 d$ states with respect to the Fermi level. Various magnetic phases are obtained depending on the combination of these characteristics. The magnetic phase diagram is determined at $T=0$. We find that in the case of a large conduction electron spin polarization and the unoccupied Mn $3 d$ states lying far above the Fermi level, an RKKY-type ferromagnetic interaction is dominating. On the other hand, the antiferromagnetic superexchange becomes important in the presence of large peaks of the unoccupied Mn 3d states lying close to the Fermi energy. The resulting magnetic behavior depends on the competition of these two exchange mechanisms. The obtained results are in good correlation with the conclusions made on the basis of the Anderson $s-d$ model and with available experimental data. These findings suggest that a targeted influence on the corresponding physical quantities can provide a useful tool for the fabrication of materials with desired physical properties.

\section{ACKNOWLEDGMENT}

The financial support of Bundesministerium für Bildung und Forschung is acknowledged.

\footnotetext{
*e.sasioglu@fz-juelich.de

†'sandr@mpi-halle.de

tbruno@mpi-halle.de

${ }^{1}$ P. J. Webster and K. R. A. Ziebeck, in Alloys and Compounds of d-Elements with Main Group Elements, edited by H. R. J. Wijn, Landolt-Börnstein, New Series, Group III, Vol. 19/c, Pt 2 (Springer, Berlin, 1988).
}

${ }^{2}$ P. J. Webster, K. R. A. Ziebeck, and K.-U. Neumann, in Magnetic Properties of Metals, edited by H. R. J. Wijn, Landolt-Börnstein, New Series, Group III, Vol. 32/c (Springer, Berlin, 2001).

${ }^{3}$ R. A. de Groot, F. M. Mueller, P. G. van Engen, and K. H. J. Buschow, Phys. Rev. Lett. 50, 2024 (1983).

${ }^{4}$ I. Galanakis, P. H. Dederichs, and N. Papanikolaou, Phys. Rev. B 66, 134428 (2002); 66, 174429 (2002); I. Galanakis, Ph. 
Mavropoulos, and P. H. Dederichs, J. Phys. D 39, 765 (2006); I. Galanakis and Ph. Mavropoulos, J. Phys.: Condens. Matter 19, 315213 (2007); S. Ishida, S. Akazawa, Y. Kubo, and J. Ishida, J. Phys. F: Met. Phys. 12, 1111 (1982); S. Ishida, S. Fujii, S. Kashiwagi, and S. Asano, J. Phys. Soc. Jpn. 64, 2152 (1995).

${ }^{5}$ P. Larson, S. D. Mahanti, and M. G. Kanatzidis, Phys. Rev. B 61, 8162 (2000).

${ }^{6}$ D. Orgassa, H. Fujiwara, T. C. Schulthess, and W. H. Butler, Phys. Rev. B 60, 13237 (1999).

${ }^{7}$ S. Picozzi, A. Continenza, and A. J. Freeman, Phys. Rev. B 69, 094423 (2004); I. Galanakis, K. Özdoğan, B. Aktaş, and E. Şaşıŏlu, Appl. Phys. Lett. 89, 042502 (2006); K. Özdoğan, B. Aktaş, I. Galanakis, and E. Şaşığlu, J. Appl. Phys. 101, 073910 (2007).

${ }^{8}$ P. A. Dowben and R. Skomski, J. Appl. Phys. 93, 7948 (2003); R. Skomski and P. A. Dowben, Europhys. Lett. 58, 544 (2002).

${ }^{9}$ S. J. Hashemifar, P. Kratzer, and M. Scheffler, Phys. Rev. Lett. 94, 096402 (2005).

${ }^{10}$ K. Nagao, Y. Miura, and M. Shirai, Phys. Rev. B 73, 104447 (2006); V. N. Antonov, H. A. Dür, Yu Kucherenko, L. V. Bekenov, and A. N. Yaresko, ibid. 72, 054441 (2005).

${ }^{11}$ I. Galanakis, M. Ležaić, G. Bihlmayer, and S. Blügel, Phys. Rev. B 71, 214431 (2005); M. Ležaić, Ph. Mavropoulos, J. Enkovaara, G. Bihlmayer, and S. Blügel, Phys. Rev. Lett. 97, 026404 (2006).

${ }^{12}$ L. Chioncel, E. Arrigoni, M. I. Katsnelson, and A. I. Lichtenstein, Phys. Rev. Lett. 96, 137203 (2006); L. Chioncel, M. I. Katsnelson, R. A. de Groot, and A. I. Lichtenstein, Phys. Rev. B 68, 144425 (2003).

${ }^{13}$ J. Rusz, L. Bergqvist, J. Kudrnovský, and I. Turek, Phys. Rev. B 73, 214412 (2006); Ján Rusz, Josef Kudrnovský, and Ilja Turek, J. Magn. Magn. Mater. 310, 1654 (2007).

${ }^{14}$ M. Sargolzaei, M. Richter, K. Koepernik, I. Opahle, H. Eschrig, and I. Chaplygin, Phys. Rev. B 74, 224410 (2006).

${ }^{15}$ R. Weht and W. E. Pickett, Phys. Rev. B 60, 13006 (1999); T. Jeong, Ruben Weht, and W. E. Pickett, ibid. 71, 184103 (2005); I. Galanakis, K. Özdoğan, E. Şaşığlu, and B. Aktaş, ibid. 75, 172405 (2007); 75, 092407 (2007).

${ }^{16}$ J. Q. Xie, J. W. Dong, J. Lu, C. J. Palmstrøm, and S. McKernan, Appl. Phys. Lett. 79, 1003 (2001); M. Kurfiss and R. Anton, J. Alloys Compd. 361, 36 (2003).

${ }^{17}$ M. N. Kirillova, A. A. Makhnev, E. I. Shreder, V. P. Dyakina, and N. B. Gorina, Phys. Status Solidi B 187, 231 (1995); K. E. H. M. Hanssen and P. E. Mijnarends, Phys. Rev. B 34, 5009 (1986); K. E. H. M. Hanssen, P. E. Mijnarends, L. P. L. M. Rabou, and K. H. J. Buschow, ibid. 42, 1533 (1990).

${ }^{18}$ S. Kämmerer, A. Thomas, A. Hütten, and G. Reiss, Appl. Phys. Lett. 85, 79 (2004); S. Okamura, R. Goto, S. Sugimoto, N. Tezuka, and K. Inomata, J. Appl. Phys. 96, 6561 (2004); E. Girgis, P. Bach, C. Rüster, C. Gould, G. Schmidt, and L. W. Molenkamp, Appl. Phys. Lett. 86, 142503 (2005).

${ }^{19}$ B. Balke, G. H. Fecher, H. C. Kandpal, C. Felser, K. Kobayashi, E. Ikenaga, J.-J. Kim, and S. Ueda, Phys. Rev. B 74, 104405 (2006); H. C. Kandpal, G. H. Fecher, C. Felser, and G. Schönhense, ibid. 73, 094422 (2006); S. Wurmehl, G. H. Fecher, H. C. Kandpal, V. Ksenofontov, C. Felser, and H. J. Lin, Appl. Phys. Lett. 88, 032503 (2006).

${ }^{20}$ I. Takeuchi, O. O. Famodu, J. C. Read, M. A. Aronova, K.-S. Chang, C. Craciunescu, S. E. Lofland, M. Wuttig, F. C. Wellstood, L. Knauss, and A. Orozco, Nat. Mater. 2, 180 (2003).
${ }^{21}$ T. Krenke, E. Duman, M. Acet, E. F. Wassermann, X. Moya, L. Mañosa, and A. Planes, Nat. Mater. 4, 450 (2005).

${ }^{22}$ J. Enkovaara, A. Ayuela, J. Jalkanen, L. Nordström, and R. M. Nieminen, Phys. Rev. B 67, 054417 (2003).

${ }^{23}$ A. T. Zayak, P. Entel, K. M. Rabe, W. A. Adeagbo, and M. Acet, Phys. Rev. B 72, 054113 (2005); T. Büsgen, J. Feydt, R. Hassdorf, S. Thienhaus, M. Moske, M. Boese, A. Zayak, and P. Entel, ibid. 70, 014111 (2004); A. T. Zayak, P. Entel, J. Enkovaara, A. Ayuela, and R. M. Nieminen, ibid. 68, 132402 (2003); P. Entel, V. D. Buchelnikov, V. V. Khovailo, A. T. Zayak, W. A. Adeagbo, M. E. Gruner, H. C. Herper, and E. F. Wassermann, J. Phys. D 39, 865 (2006).

${ }^{24}$ Thorsten Krenke, Mehmet Acet, Eberhard F. Wassermann, Xavier Moya, Lluís Mañosa, and Antoni Planes, Phys. Rev. B 73, 174413 (2006); 72, 014412 (2005).

${ }^{25}$ Harsh Deep Chopra, Chunhai Ji, and V. V. Kokorin, Phys. Rev. B 61, R14913 (2000); Matthew R. Sullivan, Ashish A. Shah, and Harsh Deep Chopra, ibid. 70, 094428 (2004).

${ }^{26}$ Thorsten Krenke, Eyüp Duman, Mehmet Acet, Eberhard F. Wassermann, Xavier Moya, Lluís Mañosa, Antoni Planes, Emmanuelle Suard, and Bachir Ouladdiaf, Phys. Rev. B 75, 104414 (2007); Jordi Marcos, Lluís Mañosa, Antoni Planes, Fèlix Casanova, Xavier Batlle, and Amílcar Labarta, ibid. 68, 094401 (2003).

${ }^{27}$ K. G. Sandeman, R. Daou, S. Özcan, J. H. Durrell, N. D. Mathur, and D. J. Fray, Phys. Rev. B 74, 224436 (2006).

${ }^{28}$ M. Pasquale, C. P. Sasso, L. Giudici, T. Lograsso, and D. Schlagel, Appl. Phys. Lett. 91, 131904 (2007); Mahmud Khan, Naushad Ali, and Shane Stadler, J. Appl. Phys. 101, 053919 (2007).

${ }^{29}$ Z. D. Han, D. H. Wang, C. L. Zhang, H. C. Xuan, B. X. Gu, and Y. W. Du, Appl. Phys. Lett. 90, 042507 (2007); Shane Stadler, Mahmud Khan, Joseph Mitchell, Naushad Ali, Angelo M. Gomes, Igor Dubenko, Armando Y. Takeuchi, and Alberto P. Guimarães, ibid. 88, 192511 (2006).

${ }^{30}$ E. Frikkee, J. Phys. F: Met. Phys. 8, L141 (1978).

${ }^{31}$ K. R. A. Ziebeck, P. J. Webster, P. J. Brown, and J. A. C. Bland, J. Magn. Magn. Mater. 24, 258 (1981).

${ }^{32}$ S. Pologmann, T. Schlatholter, J. Braun, M. Neumann, Y. M. Yarmoshenko, M. Yablonskikh, E. I. Shreder, E. Z. Kurmaev, A. Wrona, and A. Slebarski, Phys. Rev. B 60, 6428 (1999); M. V. Yablonskikh, J. Braun, M. T. Kuchel, A. V. Postnikov, J. D. Denlinger, E. I. Shreder, Y. M. Yarmoshenko, M. Neumann, and A. Moewes, ibid. 74, 085103 (2006).

${ }^{33}$ C. Jiang, M. Venkatesan, and J. M. D. Coey, Solid State Commun. 118, 513 (2001).

${ }^{34}$ J. Boeuf, C. Pfleiderer, and A. Faiszt, Phys. Rev. B 74, 024428 (2006).

${ }^{35}$ K. R. A. Ziebeck and P. J. Webster, J. Phys. F: Met. Phys. 5, 1756 (1975).

${ }^{36}$ T. Eriksson, L. Bergqvist, T. Burkert, S. Felton, R. Tellgren, P. Nordblad, O. Eriksson, and Y. Andersson, Phys. Rev. B 71, 174420 (2005).

${ }^{37}$ Y. Noda and Y. Ishikawa, J. Phys. Soc. Jpn. 40, 690 (1976); 40, 699 (1976).

${ }^{38}$ K. Tajima, Y. Ishikawa, P. J. Webster, M. V. Stringfellow, D. Tocchetti, and K. R. A. Ziebeck, J. Phys. Soc. Jpn. 43, 483 (1977).

${ }^{39}$ M. A. Ruderman and C. Kittel, Phys. Rev. 96, 99 (1954); T. Kasuya, Prog. Theor. Phys. 16, 45 (1956); K. Yosida, Phys. Rev. 
106, 893 (1957).

${ }^{40}$ P. W. Anderson, Phys. Rev. 124, 41 (1961).

${ }^{41}$ P. W. Anderson and S. Alexander, Phys. Rev. 133, A1594 (1964).

${ }^{42}$ B. Caroli and A. Blandin, J. Phys. Chem. Solids 27, 503 (1966); B. Caroli, ibid. 28, 1427 (1967).

${ }^{43}$ G. Malmström, D. J. W. Geldart, and C. Blomberg, J. Phys. F: Met. Phys. 6, 233 (1976); 6, 1953 (1976).

${ }^{44}$ D. C. Price, J. Phys. F: Met. Phys. 8, 933 (1978).

${ }^{45}$ G. Malmström and D. J. W. Geldart, J. Phys. F: Met. Phys. 8, L17 (1978).

${ }^{46}$ J. Kübler, A. R. Williams, and C. B. Sommers, Phys. Rev. B 28 , 1745 (1983).

${ }^{47}$ E. Şaşıŏlu, L. M. Sandratskii, and P. Bruno, Phys. Rev. B 70, 024427 (2004).

${ }^{48}$ E. Şaşığlu, L. M. Sandratskii, and P. Bruno, Phys. Rev. B 71, 214412 (2005).

${ }^{49}$ E. Şaşığlu, L. M. Sandratskii, and P. Bruno, J. Phys.: Condens. Matter 17, 995 (2005); J. Appl. Phys. 98, 063523 (2005); J. Magn. Magn. Mater. 290-291, 385 (2005); E. Şaşığlu, L. M. Sandratskii, P. Bruno, and I. Galanakis, Phys. Rev. B 72, 184415 (2005).

${ }^{50}$ E. Şaşığlu, L. M. Sandratskii, and P. Bruno, Appl. Phys. Lett. 89, 222508 (2006).

${ }^{51}$ Y. Kurtulus, R. Dronskowski, G. D. Samolyuk, and V. P. Antropov, Phys. Rev. B 71, 014425 (2005).

${ }^{52}$ L. M. Sandratskii and E. Şaşığlu, Phys. Rev. B 74, 214422 (2006); L. M. Sandratskii, R. Singer, and E. Şaşığlu, ibid. 76, 184406 (2007).

${ }^{53}$ O. N. Mryasov, J. Magn. Magn. Mater. 272-276, 800 (2004); Phase Transitions 78, 197 (2005).

${ }^{54}$ M. Fähnle, R. Singer, D. Steiauf, and V. P. Antropov, Phys. Rev. B 73, 172408 (2006).

${ }^{55}$ P. J. Webster and M. R. I. Ramadan, J. Magn. Magn. Mater. 5, 51 (1977); 13, 301 (1979).

${ }^{56}$ S. K. Ren, W. Q. Zou, J. Gao, X. L. Jiang, F. M. Zhang, and Y. W. Du, J. Magn. Magn. Mater. 288, 276 (2005).

${ }^{57}$ C. Walle, L. Offernes, and A. Kjekshus, J. Alloys Compd. 349, 105 (2003).

${ }^{58}$ P. Jena and D. J. W. Geldart, Phys. Rev. B 7, 439 (1973).

${ }^{59}$ N. Karnezos and J. A. Gardner, Phys. Rev. B 9, 3106 (1974).

${ }^{60}$ R. E. Walstedt and L. R. Walker, Phys. Rev. B 11, 3280 (1975).

${ }^{61}$ Q. Zhang and P. M. Levy, Phys. Rev. B 34, 1884 (1986).

${ }^{62}$ C. E. T. Gonçalves Da Silva and L. M. Falicov, J. Phys. C 5, 63 (1972).

${ }^{63}$ M. Acquarone and P. Monachesi, Phys. Rev. B 38, 2555 (1988).

${ }^{64}$ Y. Wang, P. M. Levy, and J. L. Fry, Phys. Rev. Lett. 65, 2732 (1990)

${ }^{65}$ Zhu-Pei Shi, Peter M. Levy, and John L. Fry, Phys. Rev. Lett. 69, 3678 (1992).

${ }^{66}$ Zhu-Pei Shi, Peter M. Levy, and John L. Fry, Phys. Rev. B 49, 15159 (1994).

${ }^{67}$ V. I. Litvinov and V. K. Dugaev, Phys. Rev. Lett. 86, 5593 (2001).

${ }^{68}$ S. Schwieger and W. Nolting, Phys. Rev. B 65, 205210 (2002).

${ }^{69}$ Victor Barzykin, Phys. Rev. B 71, 155203 (2005).

${ }^{70}$ Richard Bouzerar, Georges Bouzerar, and Timothy Ziman, Phys. Rev. B 73, 024411 (2006).

${ }^{71}$ A. R. Williams, J. Kübler, and C. D. Gelatt, Phys. Rev. B 19, 6094 (1979).

${ }^{72}$ O. K. Andersen, Phys. Rev. B 12, 3060 (1975).
${ }^{73}$ J. P. Perdew and Y. Wang, Phys. Rev. B 45, 13244 (1992).

${ }^{74}$ J. M. Schoen, Phys. Rev. 184, 858 (1969).

${ }^{75}$ N. M. Rosengaard and B. Johansson, Phys. Rev. B 55, 14975 (1997); S. V. Halilov, H. Eschrig, A. Y. Perlov, and P. M. Oppeneer, ibid. 58, 293 (1998); L. M. Sandratskii and P. Bruno, ibid. 67, 214402 (2003).

${ }^{76}$ M. Pajda, J. Kudrnovský, I. Turek, V. Drchal, and P. Bruno, Phys. Rev. B 64, 174402 (2001).

${ }^{77}$ A. Kimura, S. Suga, T. Matsushita, T. Kaneko, and T. Kanomata, Solid State Commun. 85, 901 (1993).

${ }^{78}$ H. Sato, M. Tamura, N. Happo, T. Mihara, M. Taniguchi, T. Mizokawa, A. Fujimori, and Y. Ueda, Solid State Commun. 92, 921 (1994).

${ }^{79}$ A. Kimura, S. Suga, T. Shishidou, S. Imada, T. Muro, S. Y. Park, T. Miyahara, T. Kaneko, and T. Kanomata, Phys. Rev. B 56, 6021 (1997).

${ }^{80}$ A. Oswald, R. Zeller, P. J. Braspenning, and P. H. Dederichs, J. Phys. F: Met. Phys. 15, 193 (1985); S. Khmelevskyi, J. Kudrnovský, B. L. Gyorffy, P. Mohn, V. Drchal, and P. Weinberger, Phys. Rev. B 70, 224432 (2004).

${ }^{81}$ A. I. Liechtenstein, M. I. Katsnelson, and V. A. Gubanov, J. Phys. F: Met. Phys. 14, L125 (1984); J. Magn. Magn. Mater. 67, 65 (1987).

${ }^{82}$ B. Möller and P. Wölfle, Phys. Rev. B 48, 10320 (1993); A. N. Tahvildar-Zadeh, M. Jarrell, and J. K. Freericks, ibid. 55, R3332 (1997).

${ }^{83}$ D. Meyer and W. Nolting, Eur. Phys. J. B 18, 385 (2000); S. Schwieger and W. Nolting, Phys. Rev. B 64, 144415 (2001).

${ }^{84}$ C. D. Batista, J. Bonča, and J. E. Gubernatis, Phys. Rev. Lett. 88, 187203 (2002); Phys. Rev. B 68, 214430 (2003).

${ }^{85}$ P. Fulde, J. Keller, and G. Zwicknagl, in Solid State Physics, edited by H. Ehrenreich and D. Turnbull (Academic, New York, 1990), Vol. 41, p. 1.

${ }^{86}$ R. E. Watson, S. Koide, M. Peter, and A. J. Freeman, Phys. Rev. 139, A167 (1965); R. E. Watson, A. J. Freeman, and S. Koide, Phys. Rev. 186, 625 (1969).

${ }^{87}$ J. Kübler, Phys. Rev. B 67, 220403(R) (2003).

${ }^{88}$ Mark van Schilfgaarde and O. N. Mryasov, Phys. Rev. B 63, 233205 (2001).

${ }^{89}$ L. M. Sandratskii and P. Bruno, J. Phys.: Condens. Matter 15, L585 (2003); J. Kudrnovský, V. Drchal, I. Turek, L. Bergqvist, O. Eriksson, G. Bouzerar, L. Sandratskii, and P. Bruno, ibid. 16, S5571 (2004).

${ }^{90}$ A. Sakuma, J. Phys. Soc. Jpn. 71, 2534 (2002).

${ }^{91}$ B. Sanyal, L. Bergqvist, and O. Eriksson, Phys. Rev. B 68, 054417 (2003); B. Sanyal, O. Eriksson, and C. Aron, ibid. 74, 184401 (2006).

${ }^{92}$ E. Şaşığlu, I. Galanakis, L. M. Sandratskii, and P. Bruno, J. Phys.: Condens. Matter 17, 3915 (2005).

${ }^{93}$ K. Sato, W. Schweika, P. H. Dederichs, and H. KatayamaYoshida, Phys. Rev. B 70, 201202(R) (2004); Yun Hee Chang, Chul Hong Park, Kazunori Sato, and Hiroshi KatayamaYoshida, ibid. 76, 125211 (2007).

${ }^{94}$ C. C. M. Campbell, J. Phys. F: Met. Phys. 5, 1931 (1975).

${ }^{95}$ Le D. Khoi, P. Veillet, and I. A. Campbell, J. Phys. F: Met. Phys. 8, 1811 (1978).

${ }^{96}$ E. Zukowski et al., J. Phys.: Condens. Matter 9, 10993 (1997).

${ }^{97}$ A. Deb, N. Hiraoka, M. Itou, Y. Sakurai, M. Onodera, and N. Sakai, Phys. Rev. B 63, 205115 (2001).

${ }^{98}$ T. Kanomata, K. Shirakawa, and T. Kaneko, J. Magn. Magn. 
Mater. 65, 76 (1987).

${ }^{99}$ T. Kaneko, K. Shirakawa, T. Kanomata, K. Watanabe, and H. Masumoto, J. Magn. Magn. Mater. 54, 933 (1986); T. Kaneko, K. Watanabe, K. Shirakawa, and H. Masumoto, ibid. 31, 79
(1983); K. Shirakawa, T. Kanomata, and T. Kaneko, ibid. 70, 421 (1987)

${ }^{100}$ A. G. Gavriliuk, G. N. Stepanov, V. A. Sidorov, and S. M. Irkaev, J. Appl. Phys. 79, 2609 (1995). 